\title{
MATHEMATICAL MODELS FOR LASER-PLASMA INTERACTION
}

\author{
RÉMi SENTIS ${ }^{1}$
}

\begin{abstract}
We address here mathematical models related to the Laser-Plasma Interaction. After a simplified introduction to the physical background concerning the modelling of the laser propagation and its interaction with a plasma, we recall some classical results about the geometrical optics in plasmas. Then we deal with the well known paraxial approximation of the solution of the Maxwell equation; we state a coupling model between the plasma hydrodynamics and the laser propagation. Lastly, we consider the coupling with the ion acoustic waves which has to be taken into account to model the so called Brillouin instability. Here, besides the macroscopic density and the velocity of the plasma, one has to handle the space-time envelope of the main laser wave, the space-time envelope of the stimulated Brillouin backscattered laser wave and the space envelope of the Brillouin ion acoustic waves. Numerical methods are also described to deal with the paraxial model and the three-wave coupling system related to the Brillouin instability.
\end{abstract}

Mathematics Subject Classification. 35Q55, 35Q60, 65M06, 34E20, 82D10.

Received: June 25, 2004.

\section{INTRODUCTION}

This paper has two goals. First, it is a review for readers involved in applied mathematics in order to describe some models used in laser-plasma interaction and to show the links between different models; we also try to give precisely the assumptions which allow to switch from one model to another. Secondly, we give some features aiming at understanding the mathematical properties of these models, we emphasize specially the boundary conditions for different models and we give some enlightments on the methods for solving them numerically. Of course, we only deal with very few models used in laser-plasma interaction which is a very intense research area in physics. For instance we do not consider any kinetic effects in the plasma and any phenomena related to ultra-high laser intensity or ultra-short laser pulse (we neither consider any relativity effect); electron population is always assumed to be at local thermodynamical equilibrium (the distribution function is always a Maxwell distribution).

In this kind of problems, some typical lengths and some typical speeds occur which are related to different physical phenomena and which range over several orders of magnitude.

Considering the spatial variable, four scales are relevant:

i) the typical length $L_{p l}$ of variation of the mean electron density of the plasma;

\footnotetext{
Keywords and phrases. Euler-Maxwell system, numerical plasma simulation, geometrical optics, paraxial approximation, Schrödinger equation, three-wave coupling system, Brillouin instability.

${ }^{1}$ CEA/Bruyeres, B.P. 12, 91680 Bruyères-le-Chatel, France. remi.sentis@cea.fr
} 
ii) the typical length $L_{l}$ of variation of the amplitude of the laser intensity, $2 \pi L_{l}$ is in the order of the width of the speckles (which are hot spots of light in the laser beam); $L_{l}$ also the length of variation of the local perturbation of the electron density;

iii) the laser wave length in the vacuum $2 \pi / k_{0}$;

iv) the Debye length $\lambda_{D}$ which is a characteristic distance of shielding in the plasma (the plasma electrons shield out a discrete electric charge at this distance).

Generally one has:

$$
\lambda_{D} \ll 2 \pi / k_{0} \ll 2 \pi L_{l} \ll L_{p l} .
$$

For example, for the high power intensity lasers (for National Ignition Facility in the USA and Laser Mega Joules facility in France) the wave length is equal to a fraction of one micron, $L_{l}$ is typically of order of one micron (then $k_{0} L_{l} \approx 10$ ) and $L_{p l}$ is larger than 100 microns. For a typical plasma, the ion temperature is in the order of $10^{7}$ Kelvin and electron temperature of $4 \times 10^{7} \mathrm{Kelvin}$, and the Debye length in the interesting region for laser interaction is smaller than 0.04 microns. On the other hand, compared to the speed of light, the electron thermal speed (proportional to the square root of the temperature) and the ion sound speed are very small: the electron thermal speed is in the order of 5 or 7 percent of $c$ and the ion sound speed in the order of $10^{-3}$ of $c$.

Several physical phenomena may occur in these plasmas: since the refraction index of the plasma depends on its density, there is refraction of light at the macroscopic level (characterized by $L_{p l}$ ); there is also refraction of light at the scale of the width of the speckles (characterized by $L_{l}$ ) which produces a self-focusing of these speckles; there is absorption of the laser energy by the plasma; at the scale of the width of the speckles, the diffraction of the laser light has to be taken into account. Moreover, the coupling of the laser waves and the plasma waves can produce instabilities: the main ones are called the Raman instability and the Brillouin instability. They are characterized by the generation of backscattered laser waves and electron or ion plasma waves.

The first section may be considered as a very quick and simplified introduction to the physical background concerning the modelling of the laser propagation and its interaction with a plasma. We start from something like a first principle system which consists in the electron Euler equations, the ion Euler equations (for the plasma) and the Maxwell equations (for the laser). Since the transverse parts of the electromagnetic fields are oscillating at very high frequency (the laser pulsation $\omega_{0}$ is fixed in the whole paper), a time envelope of these electromagnetic quantities and of the transverse electric current is made by a classical way. Then we point out, at the end of this section, that two broad categories of models may be derived. The first ones correspond to the case where the plasma behiavor is simulated mainly by a ion fluid model. In the second ones, the coupling of the laser with the Langmuir electron waves is taken into account (notice that in that case the time scale of the process is very small compared to the hydrodynamical time scale); we do not deal with these second kind of models in the present paper.

The sequel of the paper is organized as follows.

In Section 2, we focus on the main classical approximations made in laser-plasma interaction: we first show how the Laplace force may be reduced to a simple external force called ponderomotive force; we also make the quasi-neutrality approximation (which is one of the key concept in plasma physics, see [14]). These approximations lead to the so called basic time envelope model (see (25)-(27)), where the unknown functions are the time envelope of the transverse laser field, the density and the ion velocity of the plasma. This system consists in a Schrödinger equation coupled with the ion Euler equation. This model is stated in [1,23] for example. From a numerical point of view, since it is necessary to have at least eight cells per wave length to get accurate results, it is very difficult to use this model for realistic simulations. Nethertheless, for 1D simulations or 2D simulations on small spatial domain, some numerical simulations based on this model has been performed (see the previous references and [26]). Notice that with massively parallel computer architectures, it is now possible to handle this model with larger spatial domain (see [19] for example).

In Section 3, we use the Wenzel-Kramer-Brillouin (W.K.B.) approximation in order to derive the classical geometrical optics model. Strictly speaking this model does not fit in the framework of laser-plasma interaction 
since there is no interaction of the laser onto the plasma behavior (except the absorption process which heats the plasma), but it is interesting to bring together this model and the one presented in Section 4 which are both based on the W.K.B. approximation. The laser modelling is performed by the spatial and temporal envelope of the transverse laser field. The derivation of this model has been well known for a long time; see $[25,29]$ for example and [31] for a more recent presentation. For a rigorous presentation of the geometrical optics approximation in the case of a source located on a manifold, see $[5,13]$. For a numerical treatment by a classical ray tracing method, see for example [43]; and by Eulerian methods see [4,6,7,44]. The geometrical optics model has been also intensively used for a long time in acoustics problems, see for example [30].

In Section 4, a paraxial model is obtained by performing one order further the asymptotic expansion corresponding to the W.K.B. approximation. It is done in a general framework where there is an incident angle between the direction of the incoming laser wave and the gradient of the density of the plasma. If the incident angle is zero, one recovers the classical paraxial equation. We sketch briefly in Section 4.4 some features concerning the numerical methods for this model. Numerous paraxial models of this type have been used for a long time in laser-plasma interaction, see for example [3,10,22,40]. For the introduction of the paraxial approximation with a non zero incident angle see [20]. This paraxial approximation is also used for modelling other physical phenomena such as the propagation of submarine acoustic waves -the corresponding equation is known as parabolic equation- (see $[33,36,45]$ ); or the propagation of free surface waves subject to gravity and capillarity (see [11]).

In Section 5, we consider the coupling with the ion acoustic waves in order to take into account the Brillouin instability which creates the so called stimulated Brillouin backscattered laser wave. Here, besides the macroscopic density and the velocity of the plasma, one has to handle the spatial and temporal envelope of the main and backscattered transverse laser fields and the space envelope of the ion acoustic waves generated by the Brillouin instability. We explain how to derive the three-wave coupling model which is the most popular for modelling the Brillouin instability (it is also known as the standard decay model). The coupling of a paraxial model with a model of Brillouin backscattered laser wave has been performed for a long time but it is quite tricky, see $[9,34,39]$. For a physical introduction to phenomenology of the standard three-wave coupling model see for example [35]. The end of this section is devoted to the numerical treatment of this model.

The four first sections may be considered as a review of previous applied mathematics papers, the last section is more original in the applied mathematics area.

\section{ON THE LASER MODELLing IN A PLASMA}

We first consider the Euler-Maxwell system, where the laser modelling is very accurate and the plasma behavior is somehow simplified because the ions and electrons are considered in a fluid description and all the kinetic effects are neglected.

\subsection{Statement of the Euler-Maxwell system}

Let us denote:

$q_{e}, m_{e}$, the charge and the mass of the electrons;

$Z, m_{I}$, the ionization level and the mass of the ions;

$\omega_{0}$, the laser pulsation;

$c$, the speed of light;

$k_{0}=\omega_{0} c^{-1}$, the wave number of the laser in the vacuum;

$\varepsilon_{0}$, the vacuum permeability;

$N_{c}$, the critical density defined by $\omega_{0}^{2}=N_{c} q_{e}^{2}\left(\varepsilon_{0} m_{e}\right)^{-1}$;

$\nu_{e i}$, the electron-ion collision frequency which is proportional to the ion density and depends on the electron temperature, it may be assumed generally to be equal to the ion density times a constant (or a function which is constant in the simulation domain we consider). 
For describing the plasma, we introduce $N_{I}$ and $\mathbf{U}$ the ion density and velocity; $N_{e}$ and $\mathbf{U}_{e}^{\text {tot }}$ the electron density and velocity; $P_{I}$ and $P_{e}$ denote the ion and electron pressures. On the other hand, the laser beam is characterized by electromagnetic fields denoted by $\mathbf{E}^{t o t}, \mathbf{B}^{t o t}$. In the sequel, the wave length $2 \pi k_{0}^{-1}$ and the laser pulsation $\omega_{0}$ are fixed.

The balance equations for mass and momentum for ions read as

$$
\begin{gathered}
\frac{\partial N_{I}}{\partial t}+\nabla \cdot\left(N_{I} \mathbf{U}\right)=0 \\
\frac{\partial}{\partial t}\left(N_{I} \mathbf{U}\right)+\nabla \cdot\left(N_{I} \mathbf{U} \mathbf{U}\right)+\frac{1}{m_{I}} \nabla P_{I}=\frac{q_{e} Z}{m_{I}}\left(N_{I} \mathbf{E}^{t o t}+N_{I} \mathbf{U} \times \mathbf{B}^{t o t}\right)-\nu_{e i} N_{e} \frac{m_{e}}{m_{I}}\left(\mathbf{U}-\mathbf{U}_{e}^{t o t}\right),
\end{gathered}
$$

and, for electrons,

$$
\begin{gathered}
\frac{\partial N_{e}}{\partial t}+\nabla \cdot\left(N_{e} \mathbf{U}_{e}^{t o t}\right)=0 \\
\frac{\partial}{\partial t}\left(N_{e} \mathbf{U}_{e}^{t o t}\right)+\nabla \cdot\left(N_{e} \mathbf{U}_{e}^{t o t} \mathbf{U}_{e}^{t o t}\right)+\frac{1}{m_{e}} \nabla P_{e}=-\frac{q_{e}}{m_{e}}\left(N_{e} \mathbf{E}^{t o t}+N_{e} \mathbf{U}_{e}^{t o t} \times \mathbf{B}^{t o t}\right)+\nu_{e i} N_{e}\left(\mathbf{U}-\mathbf{U}_{e}^{t o t}\right) .
\end{gathered}
$$

The spatial variable is denoted by $x \in \mathbf{R}^{d}$ where $d=3$ (for some technical points, we will assume that $d=2$ ). As usual, for any vector $\mathbf{W}$, we denote by $\mathbf{W} \mathbf{W}$ the corresponding tensor and by $\nabla$. the divergence of a vector or a tensor. We denote by $\overline{\overline{1}}$ the unit tensor in $\mathbf{R}^{d}$.

For the sake of simplicity, one assumes here that the ion behavior is adiabatic. One assumes also that the electron temperature is given; as a matter of fact it is governed by a classical energy balance equation where electron thermal conduction occurs together with a source term coming from the absoprtion of the laser by the plasma (corresponding to the term $\nu_{e i} \partial \mathbf{E}^{r} / \partial t$ in equation (15) below).

Then, there exist positive constants $p_{c I}, \gamma_{I}$ (where $1<\gamma_{I} \leq 3$ ) and a given positive function $T_{e}$ such that

$$
P_{e}=T_{e} N_{e}, \quad P_{I}=p_{c I} N_{I}^{\gamma I} .
$$

If one defines the total electric current $\mathbf{J}^{\text {tot }}$ by

$$
\mathbf{J}^{t o t}=q_{e} Z N_{I} \mathbf{U}-q_{e} N_{e} \mathbf{U}_{e}^{t o t}
$$

the electromagnetic fields $\mathbf{E}^{t o t}, \mathbf{B}^{\text {tot }}$ satisfy the full Maxwell system

$$
\begin{aligned}
\frac{\partial}{\partial t} \mathbf{E}^{t o t}-c^{2} \operatorname{curl} \mathbf{B}^{t o t}+\varepsilon_{0}^{-1} \mathbf{J}^{t o t} & =0 \\
\frac{\partial}{\partial t} \mathbf{B}^{t o t}+\operatorname{curl} \mathbf{E}^{t o t} & =0
\end{aligned}
$$

with Poisson equation and Gauss relation which read as

$$
\nabla \cdot \mathbf{E}^{t o t}+\varepsilon_{0}^{-1} q_{e}\left(Z N_{I}-N_{e}\right)=0, \quad \nabla \cdot \mathbf{B}^{t o t}=0 .
$$

According to the relations (1),(3), one knows that $q_{e} \frac{\partial}{\partial t}\left(Z N_{I}-N_{e}\right)+\nabla . \mathbf{J}^{\text {tot }}=0$, then the Maxwell equations imply that

$$
\frac{\partial}{\partial t}\left(\nabla \cdot \mathbf{E}^{t o t}\right)=-\varepsilon_{0}^{-1} \nabla . \mathbf{J}^{t o t}=-\varepsilon_{0}^{-1} q_{e} \frac{\partial}{\partial t}\left(Z N_{I}-N_{e}\right),
$$

and we see that if the Poisson equation (7) holds at initial time, it always holds.

Remark (conservation of global momentum). According to the Maxwell equations, one checks that the electromagnetic momentum $\varepsilon_{0} \mathbf{E}^{t o t} \times \mathbf{B}^{\text {tot }}$ satisfies

$$
\frac{\partial}{\partial t}\left(\mathbf{E}^{t o t} \times \mathbf{B}^{t o t}\right)-c^{2}\left(\operatorname{curl} \mathbf{B}^{t o t}\right) \times \mathbf{B}^{t o t}-\left(\operatorname{curl} \mathbf{E}^{t o t}\right) \times \mathbf{E}^{t o t}+\varepsilon_{0}^{-1} \mathbf{J}^{t o t} \times \mathbf{B}^{t o t}=0 .
$$


Recall that for any vector $\mathbf{A}$, we have

$$
(\operatorname{curl} \mathbf{A}) \times \mathbf{A}=(\mathbf{A} \cdot \nabla) \mathbf{A}-\frac{1}{2} \nabla\left(|\mathbf{A}|^{2}\right)=\nabla \cdot\left(\mathbf{A} \mathbf{A}-\frac{1}{2} \overline{\overline{\mathbf{1}}}|\mathbf{A}|^{2}\right)-\mathbf{A}(\nabla . \mathbf{A}),
$$

thus if we define the tensor

$$
\overline{\bar{S}}=-\mathbf{E}^{t o t} \mathbf{E}^{t o t}-c^{2} \mathbf{B}^{t o t} \mathbf{B}^{t o t}+\frac{1}{2} \overline{\overline{\mathbf{1}}}\left(\left|\mathbf{E}^{t o t}\right|^{2}+c^{2}\left|\mathbf{B}^{t o t}\right|^{2}\right)
$$

we see that

$$
\frac{\partial}{\partial t}\left(\mathbf{E}^{t o t} \times \mathbf{B}^{t o t}\right)+\nabla \overline{\bar{S}}+\varepsilon_{0}^{-1} q_{e} \mathbf{E}^{t o t}\left(Z N_{I}-N_{e}\right)+\varepsilon_{0}^{-1} \mathbf{J}^{t o t} \times \mathbf{B}^{t o t}=0,
$$

therefore, we get a classical result for the conservation of global momentum

$$
\frac{\partial}{\partial t}\left[\varepsilon_{0}\left(\mathbf{E}^{t o t} \times \mathbf{B}^{t o t}\right)+m_{e} N_{e} \mathbf{U}_{e}^{t o t}+m_{I} N_{I} \mathbf{U}\right]+\nabla \cdot\left(\varepsilon_{0} \overline{\bar{S}}+m_{e} N_{e} \mathbf{U}_{e}^{t o t} \mathbf{U}_{e}^{t o t}+m_{I} N_{I} \mathbf{U U}\right)+\nabla\left(P_{I}+P_{e}\right)=0
$$

(see for instance [18], Chap. 1, see also [27]).

\subsection{Transverse/longitudinal decomposition and time envelope}

The previous model describes the phenomena at the time scale of the laser frequency $\omega_{0}$ and usually one uses time envelope of the transverse part of electromagnetic field which allows to work at a larger time scale than the one characterized by the laser frequency.

\subsubsection{Decomposition of the electromagnetic fields and electric current}

Generally, one can assume that the behavior of the ion density corresponds to a two-dimensional flow, that is to say there is an invariance direction $\mathbf{e}_{t r}$ which is fixed from now on; as a matter of fact, this direction is orthogonal to the gradient of the density and the travel direction of the laser at the boundary of the simulation domain. For the sake of simplicity, we assume that the polarization of the laser wave is linear and that it is the $s$-polarization, that is to say the laser wave travels in a direction orthogonal to $\mathbf{e}_{t r}$ and the oscillating part of the electric field is parallel to $\mathbf{e}_{t r}$.

So we make the following decomposition of the electromagnetic fields into a transverse part (orthogonal to the plane of propagation) and a longitudinal part; we state

$$
\mathbf{E}^{t o t}=\mathbf{E}^{r}+\mathbf{E}^{s}, \quad \mathbf{B}^{t o t}=\mathbf{B}^{r}
$$

The superscript.$^{r}$ stands for the rapidly oscillating parts. The field $\mathbf{E}^{s}$ is also called electrostatic field; the magnetostatic field $\mathbf{B}^{s}$ is always neglected.

Moreover, we decompose also the electron velocity into two parts

$$
\mathbf{U}_{e}^{t o t}=\mathbf{U}_{e}^{r}+\mathbf{U}_{e}^{s}
$$

where $\mathbf{U}_{e}^{r}$ is parallel to $\mathbf{e}_{t r}$ and $\mathbf{U}_{e}^{s}$ is orthogonal to $\mathbf{e}_{t r}$.

We also decompose the electric current into two parts (longitudinal and transverse). Since the mass ratio between ion and electron is large, the ion velocity may be considered as negligable with respect to the electron one and there is no ion contribution to the electric transverse current.

$$
\mathbf{J}^{t o t}=\mathbf{J}^{r}+\mathbf{J}^{s}, \quad \mathbf{J}^{r}=-q_{e} N_{e} \mathbf{U}_{e}^{r}, \quad \mathbf{J}^{s}=q_{e} Z N_{I} \mathbf{U}-q_{e} N_{e} \mathbf{U}_{e}^{s} .
$$




\subsubsection{The transverse fields}

According to (3), (4), the electron momentum equation reads

$$
\left(\frac{\partial}{\partial t}+\mathbf{U}_{e}^{t o t} \cdot \nabla\right) \mathbf{U}_{e}^{t o t}+\frac{1}{m_{e} N_{e}} \nabla P_{e}=-\frac{q_{e}}{m_{e}}\left(\mathbf{E}^{t o t}+\mathbf{U}_{e}^{t o t} \times \mathbf{B}^{t o t}\right)+\nu_{e i}\left(\mathbf{U}-\mathbf{U}_{e}^{t o t}\right) .
$$

Let us perform a projection of this relation onto the vector $\mathbf{e}_{t r}$; since $\mathbf{U}_{e}^{r} \cdot \nabla \mathbf{U}_{e}^{r}=0$, we get, by assuming that the electrostatic field is small compared to the transverse electron field:

$$
\left(\frac{\partial}{\partial t}+\mathbf{U}_{e}^{s} \cdot \nabla\right) \mathbf{U}_{e}^{r}+\frac{1}{m_{e} N_{e}} \nabla P_{e}+\frac{q_{e}}{m_{e}}\left(\mathbf{E}^{r}+\mathbf{U}_{e}^{r} \times \mathbf{B}^{r}\right)+\nu_{e i} \mathbf{U}_{e}^{r}=0 .
$$

Then, a crude approximation of the transverse electron velocity is the following:

$$
\frac{\partial}{\partial t} \mathbf{U}_{e}^{r}+\nu_{e i} \mathbf{U}_{e}^{r} \simeq-\frac{q_{e}}{m_{e}} \mathbf{E}^{r}
$$

It means that the electrons oscillate according to the vector $\mathbf{e}_{t r}$ at the laser frequency. This leads to the following closure for $\mathbf{J}^{r}$ :

$$
\frac{\partial}{\partial t} \mathbf{J}^{r}+\nu_{e i} \mathbf{J}^{r}=\frac{q_{e}^{2}}{m_{e}} N_{e} \mathbf{E}^{r}
$$

The fast oscillating electromagnetic fields satisfy the Maxwell equations

$$
\begin{aligned}
& \text { i) } \quad \frac{\partial}{\partial t} \mathbf{E}^{r}-c^{2} \operatorname{curl} \mathbf{B}^{r}+\varepsilon_{0}^{-1} \mathbf{J}^{r}=0, \quad \text { ii) } \quad \frac{\partial}{\partial t} \mathbf{B}^{r}+\operatorname{curl} \mathbf{E}^{r}=0, \\
& \text { iii } \left.) \quad \nabla \cdot \mathbf{E}^{r}=0, \quad i v\right) \quad \nabla \cdot \mathbf{B}^{r}=0 .
\end{aligned}
$$

Thus, taking the time derivative of the two first equations, we get the classical wave equation

$$
\frac{\partial^{2}}{\partial t^{2}} \mathbf{E}^{r}-c^{2} \Delta \mathbf{E}^{r}=-\varepsilon_{0}^{-1} \frac{\partial}{\partial t} \mathbf{J}^{r}
$$

Strictly speaking, we have to consider this wave equation coupled to (11). If $\nu_{e i}$ was zero, then we would obtain a simple expression for the right hand side. If it is not zero, we have to find a closure for the term $\frac{\partial}{\partial t} \mathbf{J}^{r}$. To do this, we assume that $\nu_{e i} \ll \omega_{0}$ and that the field $\mathbf{E}^{r}$ is a rapidly oscillating function at the pulsation $\omega_{0}$. According to (11), a crude approximation of $\mathbf{J}^{r}$ is

$$
\mathbf{J}^{r} \simeq-\frac{1}{\omega_{0}^{2}} \frac{\partial^{2} \mathbf{J}^{r}}{\partial t^{2}} \simeq-\frac{1}{\omega_{0}^{2}} \frac{q_{e}^{2}}{m_{e}} N_{e} \frac{\partial}{\partial t} \mathbf{E}^{r}
$$

Then,

$$
\frac{\partial}{\partial t} \mathbf{J}^{r} \simeq \frac{q_{e}^{2}}{m_{e}} N_{e}\left(\mathbf{E}^{r}+\frac{\nu_{e i}}{\omega_{0}^{2}} \frac{\partial}{\partial t} \mathbf{E}^{r}\right)
$$

and the wave equation (13) reads

$$
\frac{\partial^{2}}{\partial t^{2}} \mathbf{E}^{r}-c^{2} \Delta \mathbf{E}^{r}+\frac{q_{e}^{2}}{\varepsilon_{0} m_{e}} N_{e} \mathbf{E}^{r}+\frac{q_{e}^{2}}{\varepsilon_{0} m_{e}} N_{e} \frac{\nu_{e i}}{\omega_{0}^{2}} \frac{\partial}{\partial t} \mathbf{E}^{r}=0 .
$$




\subsubsection{Time envelope of the transverse fields}

It is convenient to introduce the potential vector $\mathbf{A}^{r}$ (associated to the Lorentz gauge) satisfying

$$
\mathbf{B}^{r}=\frac{1}{\omega_{0}} \operatorname{curl} \mathbf{A}^{r} ; \quad \mathbf{E}^{r}=-\frac{1}{\omega_{0}} \frac{\partial}{\partial t} \mathbf{A}^{r}
$$

We now introduce the time envelope of the rapidly oscillating quantities. Denoting by c.c. the complex conjugate of a quantity, we can state

$$
\mathbf{A}^{r}=\frac{1}{2} \mathbf{A} \mathrm{e}^{-i \omega_{0} t}+\text { c.c. }
$$

where $\mathbf{A}$ is a slowly time varying quantity. Then we get:

$$
\mathbf{B}^{r}=\frac{1}{2 \omega_{0}} \text { curlA } \mathbf{A} \mathrm{e}^{-i \omega_{0} t}+\text { c.c. }, \quad \mathbf{E}^{r}=\frac{i}{2} \mathbf{A} \mathrm{e}^{-i \omega_{0} t}+\text { c.c. }
$$

According to the previous closure (14), we can state

$$
\mathbf{J}^{r}=-\left(1-i \frac{\nu_{e i}}{\omega_{0}}\right) \frac{q_{e}^{2}}{m_{e} \omega_{0}} N_{e} \mathbf{A} \frac{1}{2} \mathrm{e}^{-i \omega_{0} t}+c . c .
$$

Now, since $\mathbf{A}$ is slowly time varying, one can neglect $\frac{\partial}{\partial t} \mathbf{A}$ with respect to $i \omega_{0} \mathbf{A}$ and (15) reads as:

$$
2 i \frac{1}{c} \frac{\partial}{\partial t} \mathbf{A}+\frac{1}{k_{0}} \Delta \mathbf{A}+k_{0}\left(1-\frac{N_{e}}{N_{c}}\right) \mathbf{A}+i \nu_{0} \mathbf{A}=0, \quad \text { with } \nu_{0}=\nu_{e i} \frac{N_{e}}{c N_{c}}
$$

\subsection{Comments on the modelling}

See for example the books [32] (Chaps. 3-6) and [38] (Chaps. 1 and 5) for a physical presentation of the above derivation, particularly for a justification of formula (14) of the plasma conductivity. It is also explained there that in a plasma, electron Langmuir waves may be excited or not. That corresponds to two different ways for dealing with the longitudinal field $\mathbf{E}^{s}$ and two families of models:

i) one takes into account the electron Langmuir waves. Then one has to state wave equations for the electron density $N_{e}$ and for the longitudinal field $\mathbf{E}^{s}$. These models are necessary if one wants to handle the Raman instabilities. See for example [32] (Chaps. 6, 7) or [38]. These models are related to the Zakharov models [46]. For a mathematical approach see for instance [15, 16,37].

ii) one ignores the electron Langmuir waves. Under this assumption, for the determination of the electrostatic longitudinal field $\mathbf{E}^{s}$, one uses the Poisson equation and one makes the quasi-neutrality approximation (this approximation is basic in plasma physics, see [14] for example). In the following section, we derive the basic model which is crucial for a good understanding of the coupling of the laser waves and the acoustic ion waves. This model takes into account the diffraction, the refraction and the autofocusing of the laser beam; it is also relevant for dealing with the filamentation instability phenomena and the Brillouin instability phenomena.

From now on, we focus only on the second way and we neglect the coupling with the electron Langmuir waves. 


\section{A BASIC MODEL COUPLing THE LASER WAVES AND THE ION WAVES}

We now start from the model which consists in the above time envelope equation for the potential vector (18) coupled with the Euler equations for ions and electrons (1)-(4) where the transverse components of electromagnetic fields are given by (16) and the electrostatic longitudinal field $\mathbf{E}^{s}$ satisfy:

$$
\nabla \cdot \mathbf{E}^{s}+\varepsilon_{0}^{-1} q_{e}\left(Z N_{I}-N_{e}\right)=0 .
$$

This classical Poisson equation is a simple consequence of (7).

\subsection{Statement of the basic time envelope model}

We make here several approximations in order to keep only the low frequency phenomena in the Euler equations and we recall the features of the quasi-neutrality approximation which is crucial for a simple modelling of the plasma.

\subsubsection{The electrostatic field and the ponderomotive force}

First, one checks that the longitudinal component of the electron momentum equation (4) reads

$$
\begin{aligned}
\frac{\partial}{\partial t}\left(N_{e} \mathbf{U}_{e}^{s}\right)+\nabla \cdot\left(N_{e} \mathbf{U}_{e}^{s} \mathbf{U}_{e}^{s}\right)+N_{e}\left(\mathbf{U}_{e}^{r} \cdot \nabla\right) \mathbf{U}_{e}^{s}+\mathbf{U}_{e}^{s} \nabla \cdot\left(N_{e} \mathbf{U}_{e}^{r}\right) & +\frac{1}{m_{e}} \nabla P_{e}+\frac{q_{e}}{m_{e}} N_{e} \mathbf{E}^{s}= \\
& -\frac{q_{e}}{m_{e}} N_{e} \mathbf{U}_{e}^{t o t} \times \mathbf{B}^{r}+\nabla \cdot\left(N_{e} \mathbf{U}_{e}^{r} \mathbf{U}_{e}^{r}\right)+\nu_{e i} N_{e}\left(\mathbf{U}-\mathbf{U}_{e}^{s}\right) .
\end{aligned}
$$

According to the assumptions on the laser polarization and on the plasma behavior, we have $\mathbf{U}_{e}^{r}$. $\nabla N_{e}=0$ and $\nabla \cdot \mathbf{U}_{e}^{r}=0$, thus $\nabla .\left(N_{e} \mathbf{U}_{e}^{r}\right)=0$. In the same way, $\left(\mathbf{U}_{e}^{r} \cdot \nabla\right) \mathbf{U}_{e}^{s}=0$. Moreover, by assuming that $\left|\mathbf{U}_{e}^{s}\right|$ is negligible with respect to $T_{e} / m_{e}$, one keeps only the following relation:

$$
\frac{1}{m_{e} N_{e}} \nabla P_{e}+\frac{q_{e}}{m_{e}} \mathbf{E}^{s}=\left\langle-\frac{q_{e}}{m_{e}} \mathbf{U}_{e}^{r} \times \mathbf{B}^{r}-\nabla \cdot\left(\mathbf{U}_{e}^{r} \mathbf{U}_{e}^{r}\right)\right\rangle,
$$

where we denote the low frequency component by the bracket $\langle$.$\rangle that is to say the time integral over a laser$ period $\frac{2 \pi}{\omega_{0}}$.

We now have to evaluate a closure for the right hand side of (20); this expression corresponds to the so-called ponderomotive force. To perform this, we use a very crude approximation of the transverse electron velocity

$$
\mathbf{U}_{e}^{r} \simeq \frac{q_{e}}{m_{e} \omega_{0}} \mathbf{A}^{r}
$$

then

$$
-\frac{q_{e}}{m_{e} \omega_{0}} \mathbf{U}_{e}^{r} \times \mathbf{B}^{r}-\nabla \cdot\left(\mathbf{U}_{e}^{r} \mathbf{U}_{e}^{r}\right) \simeq-\left(\frac{q_{e}}{m_{e} \omega_{0}}\right)^{2}\left(\mathbf{A}^{r} \times \operatorname{curl} \mathbf{A}^{r}+\nabla \cdot\left(\mathbf{A}^{r} \mathbf{A}^{r}\right) .\right.
$$

But, by using $\nabla \cdot \mathbf{A}^{r}=0$ and the vector identity (8), we can write

$$
\left\langle-\frac{q_{e}}{m_{e} \omega_{0}} \mathbf{U}_{e}^{r} \times \mathbf{B}^{r}-\nabla \cdot\left(\mathbf{U}_{e}^{r} \mathbf{U}_{e}^{r}\right)\right\rangle \simeq\left\langle-\frac{1}{2}\left(\frac{q_{e}}{m_{e} \omega_{0}}\right)^{2} \nabla\left|\mathbf{A}^{r}\right|^{2}\right\rangle=-\frac{q_{e}^{2}}{4 m_{e}^{2} \omega_{0}^{2}} \nabla|\mathbf{A}|^{2}
$$

Thus, we see that equation (20) can be reduced to

$$
q_{e} \mathbf{E}^{s} \simeq-\nabla\left(\gamma_{u}|\mathbf{A}|^{2}\right)-N_{e}^{-1} \nabla\left(N_{e} T_{e}\right), \quad \text { with } \gamma_{u}=\frac{q_{e}^{2}}{4 m_{e} \omega_{0}^{2}} .
$$




\subsubsection{Quasi-neutrality}

We assume here and in the sequel that the electron temperature $T_{e}$ may be considered as constant, then we see that $\mathbf{E}^{s}$ is the gradient of a potential $\varphi$ up to a multiplicative constant:

$$
q_{e} \mathbf{E}^{s} \simeq-T_{e} \nabla \varphi, \quad \varphi=\gamma_{u} T_{e}^{-1}|\mathbf{A}|^{2}+\log N_{e},
$$

and the Poisson equation (19) reads

$$
-\Delta \varphi=\frac{q_{e}^{2}}{\varepsilon_{0} T_{e}}\left(Z N_{I}-\mathrm{e}^{\varphi} \exp \left(-\frac{\gamma_{u}}{T_{e}}|\mathbf{A}|^{2}\right)\right) .
$$

Let us introduce a reference value $N_{\text {ref }}$ of the electron density and the Debye length:

$$
\lambda_{D}=\sqrt{\varepsilon_{0} T_{e} /\left(N_{r e f} q_{e}^{2}\right)} .
$$

Then the above equation becomes

$$
\begin{aligned}
-\Delta \varphi & =\frac{1}{\lambda_{D}^{2}} g\left(f-\mathrm{e}^{\varphi}\right) \\
g & =\frac{1}{N_{r e f}} \exp \left(-\frac{\gamma_{u}}{T_{e}}|\mathbf{A}|^{2}\right), \quad f=Z N_{I} \exp \left(\frac{\gamma_{u}}{T_{e}}|\mathbf{A}|^{2}\right) .
\end{aligned}
$$

Since $\lambda_{D}$ is very small compared to the characteristic lengths $L_{l}$ and $L_{p l}$, physicists claim in this case that the plasma is quasi-neutral (for a simple physical introduction see [14]). We now try to bear out this assertion. Consider equation (21) on a domain $\mathcal{D}$ supplemented with Neuman boundary conditions and assume that $f$ and $g$ are bounded and are strictly positive. It is easy to see that the problem is well posed and that the maximum principle holds for $\varphi$ :

$$
\log \min f \leq \varphi \leq \log \max f .
$$

By using a dimensionless space variable (scaled by $L_{l}$ ), a small parameter $\lambda=\lambda_{D} / L_{l}$ appears in equation (21) and we can state the following asymptotic result (for an analogous result with other boundary conditions, see $[12])$.

Lemma. Let $f$ and $g$ be strictly positive functions in $L^{\infty} \cap H^{1}(\mathcal{D})$. Let $\varphi_{\lambda}$ be the solution of

$$
-\Delta \varphi_{\lambda}=\lambda^{-2} g\left(f-\mathrm{e}^{\varphi_{\lambda}}\right),
$$

supplemented with Neuman boundary conditions. If $\lambda$ goes to 0 , then $\varphi_{\lambda}$ satisfies

$$
\begin{aligned}
\varphi_{\lambda} & \rightarrow \log f \quad \text { in } L^{2}(\mathcal{D}), \\
\nabla \varphi_{\lambda} & \rightarrow \frac{\nabla f}{f} \quad \text { in distribution sense. }
\end{aligned}
$$

Proof. Multiplying the Poisson equation by $\varphi_{\lambda}$ and integrating over $\mathcal{D}$, we see that there exists a constant $C$ such that

$$
\lambda^{2}\left\|\nabla \varphi_{\lambda}\right\|_{L^{2}}^{2}=\int g \varphi_{\lambda}\left(f-\mathrm{e}^{\varphi_{\lambda}}\right) \leq C^{2} .
$$

On the other hand, multiplying equation by $\left(f-\mathrm{e}^{\varphi_{\lambda}}\right)$ and integrating over $\mathcal{D}$, we get

$$
\lambda^{2} \int \nabla f \nabla \varphi_{\lambda}-\lambda^{2} \int\left|\nabla \varphi_{\lambda}\right|^{2} \mathrm{e}^{\varphi_{\lambda}}=\int g\left(f-\mathrm{e}^{\varphi_{\lambda}}\right)^{2},
$$


thus we get

$$
(\min g) \int\left(f-\mathrm{e}^{\varphi_{\lambda}}\right)^{2} \leq \lambda^{2}\left|\int \nabla f \nabla \varphi_{\lambda}\right| \leq C \lambda\|\nabla f\|_{L^{2}}
$$

Then the first part of the result follows. The second one is a classical outcome.

The physical interpretation of this result is the following approximation:

$$
\begin{aligned}
N_{e} & =N_{r e f} g \mathrm{e}^{\varphi} \simeq Z N_{I} \\
-\nabla \varphi & \simeq-\frac{1}{N_{I}} \nabla N_{I}-\frac{\gamma_{u}}{T_{e}} \nabla|\mathbf{A}|^{2}
\end{aligned}
$$

that is to say

$$
q_{e} \mathbf{E}^{s}=-T_{e} \nabla \varphi \simeq-\frac{1}{Z N_{I}} \nabla P_{e}-\gamma_{u} \nabla|\mathbf{A}|^{2} .
$$

The relation (22) expresses the quasi-neutrality of the plasma. On the other hand, if we consider the low frequency part of the ion momentum equation (2), we get, according to (23), (22):

$$
\frac{\partial}{\partial t}\left(N_{I} \mathbf{U}\right)+\nabla \cdot\left(N_{I} \mathbf{U U}\right)+\frac{1}{m_{I}} \nabla\left(P_{e}+P_{I}\right)=-N_{I} \gamma_{p} \nabla|\mathbf{A}|^{2},
$$

where $\gamma_{p}=\gamma_{u} m_{I}^{-1}=\frac{Z q_{e}^{2}}{4 m_{I} m_{e} \omega_{0}^{2}}\left(\right.$ we have also neglected the friction term $\left.\frac{m_{e}}{m_{I}} \nu_{e i}\left(\mathbf{U}-\mathbf{U}_{e}^{s}\right)\right)$.

\subsubsection{Statement of the model}

We can now summarize the previous model. To do this, let us introduce some classical notations. First, according to the expression of the ion pressure, we have $\nabla P_{I}=\gamma_{I} N_{I}^{\gamma_{I}-1} \nabla N_{I}$, and after introducing the acoustic sound speed $c_{s}$ given by $c_{s}=\sqrt{\left(\gamma_{I} N_{I}^{\gamma_{I}-1}+Z T_{e}\right) / m_{I}}$, we get

$$
\nabla\left(P_{e}+P_{I}\right)=m_{I} c_{s}^{2} \nabla N_{I}
$$

It is also convenient to define the dimensionless electron density:

$$
N=\frac{Z}{N_{c}} N_{I}
$$

Since the direction of the vector $\mathbf{A}$ does not appear in the model, we can denote it by a scalar $A$ in the sequel.

The unknowns of the basic time envelope model are: the laser field $A$, the density $N$ and the ion velocity $\mathbf{U}$. They satisfy

$$
\begin{aligned}
2 i \frac{1}{c} \frac{\partial}{\partial t} A+\frac{1}{k_{0}} \Delta A+k_{0}(1-N) A+i \nu_{0} A & =0 \\
\frac{\partial}{\partial t} N+\nabla(N \mathbf{U}) & =0 \\
\frac{\partial}{\partial t}(N \mathbf{U})+\nabla(N \mathbf{U} \mathbf{U})+c_{s}^{2} \nabla N & =-N \gamma_{p} \nabla|A|^{2}
\end{aligned}
$$

The coefficient $\gamma_{p}$ is constant; the sound speed $c_{s}$ is either constant or a smooth function of $N$.

Of course, one has to state boundary conditions for these three equations; particularly an inflow boundary condition has to be prescribed for the Schrödinger equation (25), see the following subsection.

This model may be considered as the basis of all the other models of this paper. 
Remark 1. On numerical simulations.

Numerical simulations based on this model are quite challenging, indeed the spatial mesh has to be very fine (at least 12 cells per wave length in each direction). On the other hand, since the light speed $c$ is very large compared to the ion sound speed $c_{s}$, it is necessary to solve (25) with an implicit method; as a matter of fact the time derivative term $\frac{2 i}{c} \frac{\partial}{\partial t} A$ may be considered as a perturbation of the Helmholtz equation

$$
\frac{1}{k_{0}} \Delta A+k_{0}(1-N) A=0
$$

Nevertheless, numerical simulations based on this model have been performed for instance in [26] for small spatial domain, see also [23,42]; for a 2D large spatial domain where one has to handle some $10^{8}$ cells and degrees of freedom, it is an interesting challenge of scientific computating (see [19]).

Remark 2. On the electron temperature.

As a matter of fact, the electron temperature $T_{e}$ is not a constant but it is weakly space varying; namely one would have to take into account the absorption of laser energy that is to say the specific internal energy $\frac{3}{2} T_{e}$ satisfies an equation of the following type:

$$
\frac{\partial}{\partial t}\left(\frac{3}{2} N T_{e}\right)+\nabla \cdot\left(\frac{3}{2} N T_{e} \mathbf{U}\right)-\nabla\left(\mathcal{F}\left(T_{e}\right)\right)=\frac{c \nu_{0}}{m_{I}}|A|^{2},
$$

where the term $\mathcal{F}\left(T_{e}\right)$ corresponds to a heat flux which is generally equal to $\nabla T_{e}$ times a thermal conductivity (for instance of Spitzer type, see [18]). But we do not consider this matter in the framework of this paper, and we assume that $T_{e}$ is at least locally constant.

\subsection{Properties of the basic time envelope model}

\subsubsection{Conservation of momentum}

Since the low frequency component of $\mathbf{E}^{s} \times \mathbf{B}^{r}$ is zero, we see, according to (9), that the low frequency component of the magnetic momentum is: $\mathbf{M}=i \frac{\varepsilon_{0}}{4 \omega_{0}} \mathbf{A} \times \operatorname{curl} \overline{\mathbf{A}}+$ c.c. $=i \frac{\varepsilon_{0}}{4 \omega_{0}}(A \nabla \bar{A}-\bar{A} \nabla A)$. Classically,

- denotes the complex conjugate. According to (25), we have

$$
\begin{aligned}
\frac{2 i}{c} \frac{\partial}{\partial t}(A \nabla \bar{A}-\bar{A} \nabla A)= & \frac{1}{k_{0}}[A \nabla(\Delta \bar{A})-(\Delta A) \nabla \bar{A}+\bar{A} \nabla(\Delta A)-(\Delta \bar{A}) \nabla A] \\
& +k_{0}[A \nabla((1-N) \bar{A})+\bar{A} \nabla((1-N) A)-(1-N)(A \nabla \bar{A}+A \nabla \bar{A})] .
\end{aligned}
$$

But it is easy to check that the following identity holds:

$$
\begin{gathered}
A \nabla(\Delta \bar{A})-(\Delta A) \nabla \bar{A}+\bar{A} \nabla(\Delta A)-(\Delta \bar{A}) \nabla A=2 \nabla \overline{\overline{S_{1}}}, \\
\overline{\overline{S_{1}}}=\overline{\overline{1}}\left(\frac{1}{2} A(\Delta \bar{A})+\frac{1}{2} \bar{A}(\Delta A)+|\nabla A|^{2}\right)-(\nabla A)(\nabla \bar{A})-(\nabla \bar{A})(\nabla A) .
\end{gathered}
$$

Thus we get:

Now, according to (27), we have:

$$
\frac{\partial}{\partial t} \mathbf{M}=\frac{\varepsilon_{0}}{4 k_{0}^{2}} \nabla \cdot \overline{\overline{S_{1}}}-\frac{\varepsilon_{0}}{4}|A|^{2} \nabla N
$$

$$
\begin{aligned}
\frac{\partial}{\partial t}\left(\mathbf{M}+m_{I} N_{I} \mathbf{U}\right)+\nabla \cdot\left(m_{I} N_{I} \mathbf{U U}\right)+\nabla\left(P_{e}+P_{I}\right) & =-N_{I} m_{I} \gamma_{p} \nabla|A|^{2}+\frac{\varepsilon_{0}}{4 k_{0}^{2}} \nabla \cdot \overline{\overline{S_{1}}}-\frac{\varepsilon_{0}}{4}|A|^{2} \nabla N \\
& =\frac{\varepsilon_{0}}{4 k_{0}^{2}} \nabla \cdot \overline{\overline{S_{1}}}-\nabla\left(m_{I} \gamma_{p}|A|^{2} N_{I}\right) .
\end{aligned}
$$


This last relation expresses a global momentum conservation. On the other hand, $m_{I} \gamma_{p}|A|^{2} N_{I}$ may be interpreted as a laser pressure.

\subsubsection{Related models}

We now describe some models derived from the basic one. We assume here that the ion sound speed $c_{s}$ is constant.

1. By neglecting the inertial term in the momentum equation and combining with the mass conservation equation, we get a linear wave equation for the plasma response:

$$
\frac{\partial^{2}}{\partial t^{2}} N-c_{s}^{2} \Delta N=\gamma_{p} \nabla\left(N \nabla|A|^{2}\right)
$$

Thus, by setting $N=N_{0}+w$, where $N_{0}$ is constant and $w$ a perturbation, and linearizing the ponderomotive term, we get the following system:

$$
\begin{aligned}
2 i \frac{1}{c} \frac{\partial}{\partial t} A+\frac{1}{k_{0}} \Delta A+k_{0}\left(1-N_{0}\right) A+i \nu_{0} A & =k_{0} w A \\
\frac{\partial^{2}}{\partial t^{2}} w-c_{s}^{2} \Delta w & =\gamma_{p} N_{0} \Delta|A|^{2} .
\end{aligned}
$$

2. Another type of model is obtained by dropping the time derivative in (25); then we have to consider

$$
\begin{aligned}
\frac{1}{k_{0}} \Delta A+k_{0}(1-N) A+i \nu_{0} A & =0, \\
\frac{\partial^{2}}{\partial t^{2}} N-c_{s}^{2} \Delta N & =\gamma_{p} \nabla\left(N \nabla|A|^{2}\right) .
\end{aligned}
$$

In some cases, when the transient phenomena have disappeared and the variation of density is very small, one can replace the last wave equation by a simpler closure $c_{s}^{2} \log N+\gamma_{p}|A|^{2}=\mathrm{C}^{\text {tot }}$ (see for instance [41]); that is to say

$$
N=N_{0} \exp \left(-\frac{\gamma_{p}|A|^{2}}{c_{s}^{2}}\right), \quad \text { with } N_{0} \text { constant }
$$

and we get the following Sine-Gordon equation:

$$
\frac{1}{k_{0}} \Delta A+k_{0}\left(1-N_{0} \exp \left(-\frac{\gamma_{p}|A|^{2}}{c_{s}^{2}}\right)\right) A+i \nu_{0} A=0
$$

\subsubsection{Boundary conditions}

Generally, one has to consider the previous models on a bounded simulation domain $\mathcal{D}$ and we must focus on the boundary conditions on one hand for (25) or (32), and on the other hand for (26)-(27) or (33) on boundary $\partial \mathcal{D}$.

a) For (25) or (32), denoting by $\mathbf{e}_{b}$ the unit vector characterizing the propagation direction of the laser, we first have to consider the lighted part of the boundary $\Gamma^{\text {in }}$ defined by

$$
\Gamma^{\text {in }}=\left\{\mathbf{x} \in \partial \mathcal{D}, \text { such that } \mathbf{e}_{b} \cdot \mathbf{n}<0\right\}, \quad \mathbf{n}, \text { the outwards normal vector. }
$$

By assuming that the density $N$ is a constant $N^{\text {in }}$ on this part of the boundary $\Gamma^{\text {in }}$, we set

$$
\mathbf{K}^{\text {in }}=\mathbf{e}_{b}\left(1-N^{\text {in }}\right)^{1 / 2},
$$


and the incident wave is assumed to be of the form $\alpha^{\mathrm{in}} \mathrm{e}^{i k_{0} \mathbf{K}^{\mathrm{in}} \mathbf{x}}$, knowing that $\alpha^{\mathrm{in}}=\alpha^{\mathrm{in}}(\mathbf{x})$ is the restriction to $\Gamma^{\text {in }}$ of a smooth function. The incoming boundary condition on $\Gamma^{\text {in }}$ reads as

$$
\left(k_{0}^{-1} \mathbf{n} \cdot \nabla+i \mathbf{K}^{\mathrm{in}} \cdot \mathbf{n}\right)\left(A-\alpha^{\mathrm{in}} \mathrm{e}^{i k_{0} \mathbf{K}^{\mathrm{in}} \mathbf{x}}\right)=0 .
$$

On the other hand, if we set $\Gamma^{\text {out }}=\partial \mathcal{D}-\left\{\Gamma^{\text {in }}\right\}$ ( where $\left.\mathbf{e}_{b} \cdot \mathbf{n} \geq \mathbf{0}\right)$, the boundary condition on $\Gamma^{\text {out }}$ reads as:

$$
\left(k_{0}^{-1} \mathbf{n} . \nabla-i \sqrt{1-N}\right) A=0 .
$$

From a numerical point of view, on $\Gamma^{\text {out }}$, we can also consider perfectly matched layers (PML) of the type described in [8]. Denote by $\mathrm{d} \Gamma$ the surface measure on $\Gamma^{\text {in }}$ and $\Gamma^{\text {out }}$.

Lemma (energy balance). Let A, solution to (32), (34), (35); then we get

$$
\begin{aligned}
\int_{\Gamma^{\text {in }}}\left(\left|\mathbf{K}^{\text {in }} \cdot \mathbf{n}\right|\left|\alpha^{\text {in }}\right|^{2}+\frac{1}{4\left|\mathbf{K}^{\text {in }} \cdot \mathbf{n}\right|}\left|k_{0}^{-1} \mathbf{n} \cdot \nabla \alpha^{\text {in }}\right|^{2}\right) & \mathrm{d} \Gamma=\int_{\Gamma^{\text {in }}} \frac{1}{4\left|\mathbf{K}^{\text {in }} \cdot \mathbf{n}\right|}\left|k_{0}^{-1} \mathbf{n} \cdot \nabla A-i \mathbf{K}^{\text {in }} \cdot \mathbf{n} A\right|^{2} \mathrm{~d} \Gamma \\
& +\int_{\Gamma^{\text {out }}} \frac{1}{4|1-N|^{1 / 2}}\left|k_{0}^{-1} \mathbf{n} \cdot \nabla A+i \sqrt{1-N} A\right|^{2} \mathrm{~d} \Gamma+\int_{D} \nu_{0}|A|^{2} \mathrm{~d} x .
\end{aligned}
$$

For the proof, one multiplies (32) by $\bar{A}$ and integrates by part.

This relation may be interpreted as energy balance: the energy flux incoming on $\Gamma^{\text {in }}$ is equal to the flux outgoing on $\Gamma^{\text {in }}$ and $\Gamma^{\text {out }}$ respectively plus the absorbed energy $\int \nu_{0}|A|^{2} \mathrm{~d} x$.

b) The problem of boundary conditions for the barotropic hydrodynamic model (26), (27) is classical at least in the subsonic case. On each part of the boundary $\partial D$, one has to give one condition: for instance one can fix the plasma density $N=N^{\text {in }}$ or one can use a transparent boundary condition, for example some PML of the type described in [8].

If one addresses the wave equation (33), analoguous boundary conditions have to be implemented.

\section{The GeOMETRICAL optics APproximation}

The geometrical optics approximation is based on a spatial envelope of the solution $A$ of the Helmholtz equation (32) supplemented with the boundary condition (34), via the W.K.B. expansion. As it was pointed out, the derivation of this model has been well known for a long time [25,29]; but even out of the framework of the scattering theory (where the propagation of the wave is perturbed by an obstacle), one knows that some difficulties arise due to the fact that a caustic surface may occur in the laser propagation.

Here we only recall some simple features of this problem. The basis assumption is that the wave length $2 \pi / k_{0}$ is small compared to the length $L_{l}$. So, we introduce a small dimensionless parameter:

$$
\epsilon=\left(k_{0} L_{l}\right)^{-1}
$$

As mentioned in the introduction, for our applications, $\epsilon$ may be in the order of 0.1 or less.

In the framework of the geometrical optics approximation, we have to assume that:

- the plasma hydrodynamics is not coupled with the laser propagation, that is to say the dimensionless plasma density $N$ is assumed to be a smooth function $N_{0}$ of the space variable, independent from the time variable (then $N(x)=N_{0}(x)$ ),

- the incoming laser intensity $\alpha^{\text {in }}$ is also independent from the time variable (this last assumption may be removed; see the remark at the end of this section).

For the sake of simplicity, we assume, up to now, that:

- the simulation domain $\mathcal{D}$ is either a strip between the two boundaries $\Gamma^{\text {in }}$ and $\Gamma^{\text {out }}$ or a parallelepiped (in that case $\alpha^{\text {in }}$ is non zero only on an edge of the domain - which is included in $\Gamma^{\mathrm{in}}$ ). 
Since there is no coupling with the plasma hydrodynamics, this section is not actually in the scope of the laser-plasma interaction, but for the sake of completeness, it is interesting to recall the principle of Geometrical Optics in our framework (indeed the same W.K.B expansion is performed here and in the following section).

With the above assumptions, equation (32) may be recasted in the following way:

$$
\epsilon L_{l} \Delta A+\frac{1}{\epsilon L_{l}}\left(1-N_{0}\right) A+i \nu_{0} A=0 .
$$

The principle of the W.K.B. expansion is to write the solution $A$ of this equation in the form

$$
A(x) \simeq\left(a_{0}(x)+\epsilon a_{1}+\ldots\right) \exp \left(i \frac{\phi(x)}{\epsilon L_{l}}\right),
$$

where $a_{0}$ and $\phi$ are slowly space varying functions. The quantities $\phi$ and $a_{0}$ are called the phase and the amplitude of the laser field.

\subsection{Eikonal equation and advection equation}

Since

$$
\exp \left(-i \frac{\phi}{\epsilon L_{l}}\right) \Delta\left(a_{0} \exp \left(i \frac{\phi}{\epsilon L_{l}}\right)\right)=\Delta a_{0}+\frac{1}{\epsilon L_{l}}\left(2 i(\nabla \phi) \cdot \nabla a_{0}+i a_{0} \Delta \phi\right)-\frac{1}{\left(\epsilon L_{l}\right)^{2}} a_{0}|\nabla \phi|^{2}
$$

we get by plugging the previous expansion in (36):

$$
\begin{aligned}
& 0=\epsilon^{-1} \frac{a_{0}}{L_{l}}\left[1-N_{0}-|\nabla \phi|^{2}\right], \\
& +\epsilon^{0}\left(i \nu_{0} a_{0}+2 i(\nabla \phi) . \nabla a_{0}+i a_{0} \Delta \phi\right)+\epsilon \ldots
\end{aligned}
$$

In order to have a non trivial value of $a_{0}$, it is necessary that the phase $\phi$ satisfies

$$
|\nabla \phi|^{2}=1-N_{0}
$$

which is called the eikonal equation.

On the incoming part of the boundary $\Gamma^{\text {in }}$, the direction of $\nabla \phi$ has to be parallel to the fixed vector $\mathbf{e}_{b}$ and to $\mathbf{K}^{\text {in }}$. Then the boundary condition for the eikonal equation is

$$
\left.\nabla \phi\right|_{\Gamma^{\text {in }}}=\mathbf{K}^{\text {in }} .
$$

Moreover, if we denote $\mathbf{K}=\nabla \phi$, we get the following advection or transport equation for the laser amplitude $a_{0}$ :

$$
\nu_{0} a_{0}+2 \mathbf{K} \cdot \nabla a_{0}+a_{0} \nabla \cdot \mathbf{K}=0 .
$$

On the incoming part of the boundary $\Gamma^{\text {in }}$, one has to prescribe the value of the intensity of the laser beam:

$$
a_{0}=\alpha^{\text {in }} .
$$

One checks that the laser energy $\left|a_{0}\right|^{2}$ satisfies:

$$
\nu_{0}\left|a_{0}\right|^{2}+\nabla \cdot\left(\mathbf{K}\left|a_{0}\right|^{2}\right)=0 .
$$


Notice that when we integrate this relation over any small subdomain $D$ of $\mathcal{D}$, we get an energy balance equation (here and in the sequel $\mathrm{d} \Gamma(x)$ denotes the restriction of the Lebesgue measure to a boundary of a subdomain):

$$
\int_{D} \nu_{0}\left|a_{0}\right|^{2} \mathrm{~d} x+\int_{\partial D} \mathbf{n} \cdot \mathbf{K}\left|a_{0}\right|^{2} \mathrm{~d} \Gamma(x)=0 .
$$

The vector $\mathbf{K}\left|a_{0}\right|^{2}$ is called the laser energy flux. One recovers here the fact that $\nu_{0}\left|a_{0}\right|^{2}$ is the density of absorbed laser energy.

In general cases, the situation is the following: there is a caustic surface $\mathcal{C}$, the solution of the eikonal equation (37) is bivalued and does not exist in the shadow region which is after the caustic surface. The two branches correspond to the direct part and return part of the beam. But, if the density $N_{0}$ is small enough, it may happen that no caustic appears in the simulation domain, that is to say there is only a mono-valued solution defined in the whole domain which corresponds to the direct beam.

The classical way to deal numerically with the Geometrical Optics model with variable index of refraction is the ray tracing method, see for example [43]; for a mathematical interpretation of this method, see [4]. In the following subsections, we briefly give some mathematical enlightments on the system (37), (38).

\subsection{Example of solutions of the eikonal equation}

Assume here that $N_{0}$ is a smooth function depending only on a single spatial coordinate $x_{1}$, which is one of the symmetry axis of domain $\mathcal{D}$. Moreover, if $\mathcal{D}$ is a strip, $\left\{x_{1}=0\right\}$ corresponds to the boundary $\Gamma^{\text {in }}$ and, if $\mathcal{D}$ is a parallelepiped, the edge of the domain $\left\{x_{1}=0\right\}$ is the one where $\alpha^{\text {in }}$ is non zero. The angle between the vector $\mathbf{e}_{b}$ and the direction $x_{1}$ is called the incidence angle.

Let us set $x=\left(x_{1}, x_{\text {orth }}\right)$ (where $\left.x_{\text {orth }} \in \mathbf{R}^{d-1}\right)$, and for any vector we denote by $\left(\bullet_{1}, \bullet_{\text {orth }}\right)$ its two components on the $x_{1}$ coordinate and on the orthogonal hyperplane. At the boundary corresponding to $x_{1}=0$, we have to set the boundary conditions:

$$
\left.\nabla \phi\right|_{1}=\mathbf{K}_{1}^{\text {in }} \quad \text { and }\left.\nabla \phi\right|_{\text {orth }}=\mathbf{K}_{\text {orth }}^{\text {in }} .
$$

Then, the two branches of the solution $\phi$ of the eikonal equation (37) may be denoted by $\phi^{-}$and $\phi^{+}$and the corresponding fields $\mathbf{K}^{-}=\nabla \phi^{-}$and $\mathbf{K}^{+}=\nabla \phi^{+}$are given by:

$$
\mathbf{K}_{\text {orth }}^{ \pm}(x)=\mathbf{K}_{\text {orth }}^{\mathrm{in}}, \quad \mathbf{K}_{1}^{ \pm}(x)=\mp\left(1-N_{0}\left(x_{1}\right)-\left|\mathbf{K}_{\text {orth }}^{\mathrm{in}}\right|^{2}\right)^{1 / 2}
$$

which is defined only for $x_{1} \in\left[0, x_{1}^{*}\right]$, where $x_{1}^{*}$ is the first value such that

$$
1-N_{0}\left(x_{1}^{*}\right)=\left|\mathbf{K}_{\text {orth }}^{\text {in }}\right|^{2} .
$$

The hyperplane corresponding to $x_{1}=x_{1}^{*}$ is the caustic surface $\mathcal{C}$. The solution of $(37)$ is bi-valued in the region before the caustic, its values $\phi^{ \pm}$are defined up an additive constant and satisfy:

$$
\begin{aligned}
& \phi^{-}\left(x_{1}, x_{\text {orth }}\right)=\mathbf{K}_{\text {orth }}^{\mathrm{in}} . x_{\text {orth }}+\int_{0}^{x_{1}}\left(1-N_{0}(s)-\left|\mathbf{K}_{\text {orth }}^{\mathrm{in}}\right|^{2}\right)^{1 / 2} \mathrm{~d} s \\
& \phi^{+}\left(x_{1}, x_{\text {orth }}\right)=\mathbf{K}_{\text {orth }}^{\mathrm{in}} . x_{\text {orth }}+\int_{x_{1}}^{x_{1}^{*}}\left(1-N_{0}(s)-\left|\mathbf{K}_{\text {orth }}^{\mathrm{in}}\right|^{2}\right)^{1 / 2} \mathrm{~d} s+C,
\end{aligned}
$$

where $C$ is a constant such that on the caustic one has $\phi^{-}\left(x_{1}, x_{\text {orth }}\right)=\phi^{+}\left(x_{1}, x_{\text {orth }}\right)$. The two branches of the solution correspond to the direct part of the beam for $\mathbf{K}_{1}^{-}($.$) and return part of the beam part of the beam$ for $\mathbf{K}_{1}^{+}($.). 


\subsection{About the advection equation}

The previous situation is generic: the eikonal equation admits a solution with two branches corresponding to two vector fields $\mathbf{K}^{-}$and $\mathbf{K}^{+}$on a domain bounded by the incoming boundary $\Gamma^{\text {in }}$ and a caustic surface denoted by $\mathcal{C}$, see [6]. Then the advection equation has also two branches: the direct one $a_{0}^{-}$corresponding to the field $\Gamma^{-}$(before reaching the caustic), and the return one $a_{0}^{+}$corresponding to the field $\Gamma^{+}$(after reaching the caustic), which satisfy:

$$
\begin{aligned}
& \nu_{0} a_{0}^{-}+2 \mathbf{K}^{-} \cdot \nabla a_{0}^{-}+a_{0}^{-} \nabla \cdot \mathbf{K}^{-}=0 \\
& \nu_{0} a_{0}^{+}+2 \mathbf{K}^{+} \cdot \nabla a_{0}^{+}+a_{0}^{+} \nabla \cdot \mathbf{K}^{+}=0 .
\end{aligned}
$$

For the direct branch laser amplitude $a_{0}^{-}$, a boundary condition has to be specified on the boundary $\Gamma^{\text {in }}$ :

$$
\left.a_{0}^{-}\right|_{\Gamma^{\text {in }}}=\alpha^{\text {in }}
$$

But it is easy to see that $\nabla \cdot \mathbf{K}^{-}(x)$ goes to $-\infty$ when $x$ goes to the caustic surface $\mathcal{C}$, therefore the amplitude $a_{0}^{-}$ converges also to infinity on $\mathcal{C}$. Moreover, one can state the energy equation for this amplitude $a_{0}^{-}$:

$$
\nu_{0}\left|a_{0}^{-}\right|^{2}+\nabla \cdot\left(\mathbf{K}^{-}\left|a_{0}^{-}\right|^{2}\right)=0
$$

If now $\nu_{0}$ is zero, one can check that the integral of $\left|a_{0}^{-}\right|^{2}$ over a tube of rays, that is to say a domain delimited by the characteristic curves corresponding to the field $\mathbf{K}^{-}$is constant. A precise definition of the boundary condition for the amplitude $a_{0}^{+}$is not possible. Indeed it should be defined on the caustic surface $\mathcal{C}$, but on this surface, the amplitudes $a_{0}^{-}, a_{0}^{+}$are infinite. So, to overcome this difficulty, one has to introduce the bicharacteristic curves of the problem, that is to say the functions $\mathbf{x}(s), \xi(s)$ depending of a positive scalar $s$ satisfying $\mathrm{d} \mathbf{x} / \mathrm{d} s=\xi, \mathrm{d} \xi / \mathrm{d} s=-\frac{1}{2} \nabla N(\mathbf{x})$, and whose initial values are parametrized by $\beta$ (belonging to the boundary $\left.\Gamma^{\text {in }}\right): \mathbf{x}_{\beta}(0)=\beta, \xi_{\beta}(0)=\mathbf{K}^{-}(\beta)$. One can see that

$$
\frac{\mathrm{d} \mathbf{x}_{\beta}}{\mathrm{d} s}=\mathbf{K}^{-}\left(\mathbf{x}_{\beta}(s)\right)
$$

On the other hand, one defines the geometrical spreading $G^{-}$of the flow $\mathbf{K}^{-}($.$) by the relation G^{-}\left(\mathbf{x}_{\beta}(s)\right)=$ $\operatorname{det}\left(\frac{\partial \mathbf{x}_{\beta}}{\partial s}(s), \frac{\partial \mathbf{x}_{\beta}}{\partial \beta}(s)\right)$ and the analogous quantity $G^{+}$for the flow $\mathbf{K}^{+}$. With classical geometric arguments, one checks:

$$
\mathbf{K}^{-} \cdot \nabla G^{-}=G^{-} \nabla \cdot \mathbf{K}^{-}, \quad \mathbf{K}^{+} \cdot \nabla G^{+}=G^{+} \nabla \cdot \mathbf{K}^{+} .
$$

Futhermore, with some technical hypothesis, one can prove that the two quantities $Z^{-}=\left|a_{0}^{-}\right|^{2} G^{-}$and $Z^{+}=$ $\left|a_{0}^{+}\right|^{2} G^{+}$are bounded solutions of the equations

$$
\begin{array}{ll}
\mathbf{K}^{-} \cdot \nabla Z^{-}+\nu Z^{-}=0, & \left.Z^{-}\right|_{\Gamma^{\text {in }}}=\left|\alpha^{\text {in }}\right|^{2} \\
\mathbf{K}^{+} \cdot \nabla Z^{+}+\nu Z^{+}=0, & \left.Z^{+}\right|_{\mathcal{C}}=\left.Z^{-}\right|_{\mathcal{C}}
\end{array}
$$

Therefore, the absorbed laser energy in a region $\mathcal{D}_{0}$ may be approximated by:

$$
\int_{\mathcal{D}_{0}^{\prime}} \nu(x)\left(\frac{Z^{-}(x)}{G^{-}(x)}+\frac{Z^{+}(x)}{G^{+}(x)}\right) \mathrm{d} x,
$$

where $\mathcal{D}_{0}^{\prime}$ is the part of $\mathcal{D}_{0}$ which is contained in the region between the boundary $\Gamma^{\text {in }}$ and the caustic surface $\mathcal{C}$. The reader is referred to [7] for details on this analysis and for a direct numerical method to deal with (38) by using (40), (41). 
Remark 1. In the case where the electron density $N_{0}$ is such that for any $x \in \mathcal{D}$, we have

$$
\operatorname{Inf}_{x \in \mathcal{D}}\left(N_{0}(x)\right)<1-\left|\mathbf{K}_{\text {orth }}^{\text {in }}\right|^{2},
$$

then, there is no caustic. The solution $\phi$ of the eikonal equation is defined smoothly on the whole simulation domain and there is only one direct propagation vector $\mathbf{K}$ corresponding to $\mathbf{K}^{-}$. The laser field $a_{0}$ is a classical solution to (38).

Remark 2. The previous approximation may be generalized if one considers the solution of equation (25) with a boundary condition with an incoming laser intensity $\alpha^{\text {in }}$ which depends slowly on the time variable; that is to say, if it is a smooth function of a reduced time variable $\epsilon t$. In this framework, the eikonal equation is the same as before and it is feasible to modify the advection equation by introducing the time dependance, that is to say, $a_{0}^{-}=a_{0}^{-}(t, x)$ depends now on $t$ and $x$, and satisfies

$$
\frac{2}{c} \frac{\partial}{\partial t} a_{0}^{-}+\nu_{0} a_{0}^{-}+2 \mathbf{K} \cdot \nabla a_{0}^{-}+a_{0}^{-} \nabla \cdot \mathbf{K}=0 .
$$

Of course, besides the boundary condition, one also has to give an initial condition $a_{0}^{-}(0,$.$) .$

\section{Paraxial approximation}

In the Geometrical Optics model, the diffraction is not taken into account; to deal with the diffraction phenomenon, it is necessary to perform a more precise approximation of the time envelope of the Maxwell equation, which is the so-called paraxial approximation. In this section we show how to switch from the basic model (25), (26), (27) to another one, where one deals with the spatial envelope of $A$.

As before, we assume that the direction $\mathbf{e}_{b}$ of the incoming laser is fixed and that the simulation domain $\mathcal{D}$ is either an half space whose boundary is the hyperplane $\Gamma^{\text {in }}$ or a parallelepiped. In order to study the paraxial approximation of the solution $A$ of equation (25), we have to make the following hypothesis.

- The density function is a sum of a smooth function at the scale of $L_{l}$ and a small perturbation; more precisely $N$ has the following form:

$$
N(t, x)=N_{0}(x)+\epsilon^{2} G(t, x) \quad \text { with } N_{0}(x)=\mathcal{N}_{0}(\epsilon x),
$$

where $\mathcal{N}_{0}$ is a smooth function (independent from $\epsilon$ ) whose gradient is uniformly bounded, and $G$ is a bounded function which may depend on the time and space variables.

- The function $N_{0}$ is such that there is no caustic in the simulation domain.

- The incoming laser field $\alpha^{\text {in }}(t)$ on the boundary $\Gamma^{\text {in }}$ (or on one edge of the parallelepiped) is slowly time varying ; more precisely we can write

$$
\alpha^{\text {in }}(t)=\widetilde{\alpha}^{\text {in }}(\epsilon t),
$$

with $\widetilde{\alpha}^{\text {in }}$ a smooth function of the reduced time variable $T=\epsilon t$.

- The absorption length $\nu_{0}^{-1}$ is large enough compared to $L_{l}$, i.e., there exists $\nu_{1}$ an absorption coefficient such that

$$
\nu_{0}=\epsilon \nu_{1} .
$$

According to assumption (42), the eikonal equation now reads as

$$
|\nabla \phi(x)|^{2}=1-\mathcal{N}_{0}(\epsilon x) .
$$

Then, we set

$$
\mathbf{K}=\nabla \phi,
$$


and it is easy to check that there exists a smooth vector function $\mathcal{K}$ whose partial derivatives are bounded, such that $\mathbf{K}(x)=\mathcal{K}(\epsilon x)$. Then we get

$$
\nabla \cdot \mathbf{K}(x)=\epsilon(\nabla \cdot \mathcal{K})(\epsilon x)=O(\epsilon) .
$$

We make the following change of variable $T=\epsilon t$, and define $\widetilde{A}(T)$ by

$$
\widetilde{A}(\epsilon t)=A(t)
$$

Then, equation (25) may be recasted in the form

$$
2 \frac{i \epsilon}{c} \frac{\partial}{\partial T} \widetilde{A}+\epsilon L_{l} \Delta \widetilde{A}+\frac{1}{\epsilon L_{l}}\left(1-N_{0}\right) \widetilde{A}-\frac{\epsilon}{L_{l}} G \widetilde{A}+i \epsilon \nu_{1} \widetilde{A}=0 .
$$

\subsection{The advection-Schrödinger equation}

We now perform an asymptotic expansion (with respect to $\epsilon$ ) of the solution $\widetilde{A}$ in a similar way as in the previous section:

$$
\widetilde{A}(T, .)=\left(a_{0}(T, .)+\epsilon a_{1}(T, .)+\epsilon^{2} \ldots\right) \exp \left(\frac{i \phi}{\epsilon L_{l}}\right),
$$

where $a_{0}, a_{1}$ are now functions depending on the reduced time variable $T$ and on the space variable.

After plugging this asymptotic expansion in the previous equation, we get

$$
\begin{aligned}
0= & \epsilon^{-1} \frac{a_{0}}{L_{l}}\left[1-N_{0}-|\mathbf{K}|^{2}\right] \\
& +i\left(2 \mathbf{K} \cdot \nabla a_{0}\right) \\
& +i\left(a_{0} \nabla \cdot \mathbf{K}+\right)+\epsilon\left[i\left(\frac{2}{c} \frac{\partial a_{0}}{\partial T}+2 \mathbf{K} \cdot \nabla a_{1}+\nu_{1} a_{0}\right)-\frac{G}{L_{l}} a_{0}+L_{l}\left(\Delta a_{0}\right)\right]+\epsilon^{2} \ldots
\end{aligned}
$$

According to the eikonal equation, the $\epsilon^{-1}$ terms are zero. The term of order zero has to be also zero, thus $a_{0}$ satisfies the simple advection equation (where the variable $T$ is a simple parameter):

$$
\text { K. } \nabla a_{0}=0, \quad a_{0}(T)=\widetilde{\alpha}^{\text {in }}(T) \quad \text { on } \Gamma^{\text {in }} .
$$

For the terms of order 1 , we get

$$
i\left(\frac{2}{c} \frac{\partial a_{0}}{\partial T}+2 \mathbf{K} \cdot \nabla a_{1}+a_{0} \frac{\nabla \cdot \mathbf{K}}{\epsilon}+\nu_{1} a_{0}\right)+L_{l}\left(\Delta a_{0}\right)-\frac{G}{L_{l}} a_{0}=0 .
$$

By combining (43) with this last equation, we get

$$
i\left(\epsilon \frac{2}{c} \frac{\partial a_{0}}{\partial T}+2 \mathbf{K} \nabla\left(a_{0}+\epsilon a_{1}\right)+a_{0} \nabla \mathbf{K}+\epsilon \nu_{1} a_{0}\right)+\epsilon L_{l}\left(\Delta a_{0}\right)-\epsilon \frac{G}{L_{l}} a_{0}=0 .
$$

Let us define the transverse gradient $\nabla_{\perp}^{K}$ (with respect to $\mathbf{K}$ ) and the transverse Laplace operator by

$$
\nabla_{\perp}^{K} \bullet=\nabla \bullet-\frac{\mathbf{K}}{|\mathbf{K}|^{2}} \mathbf{K} . \nabla \bullet, \quad \Delta_{\perp}^{K} \bullet=\nabla .\left[\left(\overline{\overline{1}}-\frac{\mathbf{K K}}{|\mathbf{K}|^{2}}\right) \nabla \bullet\right]=\nabla \cdot\left(\nabla_{\perp}^{K} \bullet\right),
$$

where, as usual, KK denotes a tensor.

According to (43), we can easily check that

$$
\nabla a_{0}=\nabla_{\perp}^{K} a_{0}, \quad \Delta a_{0}=\Delta_{\perp}^{K} a_{0}
$$


If we now set $\widetilde{E}=a_{0}+\epsilon a_{1}$, a formal calculation leads to:

$$
i\left(\epsilon \frac{2}{c} \frac{\partial \widetilde{E}}{\partial T}+2 \mathbf{K} \cdot \nabla \widetilde{E}+\widetilde{E} \nabla \cdot \mathbf{K}+\epsilon \nu_{1} \widetilde{E}\right)+\epsilon L_{l} \Delta_{\perp}^{K} \widetilde{E}-\epsilon \frac{G}{L_{l}} \widetilde{E}=O\left(\epsilon^{2}\right) .
$$

\subsubsection{Statement of the model}

Dropping the $O\left(\epsilon^{2}\right)$ and coming back to the physical variables, that is to say by defining $E(t,)=.\widetilde{E}(\epsilon t,$.$) ,$ we see heuristically that

where $E$ is solution to

$$
A(t, x) \simeq E(t, x) \mathrm{e}^{i k_{0} \phi}
$$

$$
i\left(\frac{2}{c} \frac{\partial E}{\partial t}+2 \mathbf{K} \cdot \nabla E+E(\nabla \cdot \mathbf{K})+\nu_{0} E\right)+\frac{1}{k_{0}}\left(\Delta_{\perp}^{K} E\right)-k_{0}\left(N-N_{0}\right) E=0 .
$$

It may be noticed that the time derivative term $\frac{1}{c} \frac{\partial E}{\partial t}$ is a perturbation term compared to the advection one K. $\nabla E$. Indeed the previous calculation has shown that this term is only an order $\epsilon$ term.

It is necessary to supplement equation (45) with an initial condition $E(0,$.$) . Moreover, according to the$ incoming boundary condition (34), we see that the correct boundary condition for this equation on the bound$\operatorname{ary} \Gamma^{\text {in }}$ is

$$
\left(k_{0}^{-1} \mathbf{n} \cdot \nabla_{\perp}+2 i \mathbf{K} \cdot \mathbf{n}\right)\left(E(t, .)-\alpha^{\text {in }}(t, .)\right)=0 .
$$

Notice that according to the above hypothesis, $k_{0}^{-1}$ is small compared to the characteristic length $L_{l}$, and this condition implies that the value of $E$ at the boundary is close to $\alpha^{\text {in }}$.

\section{Summary of the model}

The equation $(45),(46)$ has to be coupled with the hydrodynamic system as previously:

$$
\begin{aligned}
\frac{\partial}{\partial t}(N)+\nabla \cdot(N \mathbf{U}) & =0 \\
\frac{\partial}{\partial t}(N \mathbf{U})+\nabla \cdot(N \mathbf{U U})+c_{s}^{2} \nabla N & =-N \gamma_{p} \nabla|E|^{2} .
\end{aligned}
$$

It may also be coupled with the wave equation (29).

\subsubsection{Momentum conservation}

If $\mathbf{K}$ is not constant, it is not possible to prove a rigorous momentum conservation relation as the one presented in Section 2.2. So we assume here that $N_{0}$ is constant and thus $\mathbf{K}$ is constant. The low frequency component of the magnetic momentum is: $\mathbf{M}=\frac{\varepsilon_{0}}{4 \omega_{0}}\left(2 k_{0}|E|^{2} \mathbf{K}+i(E \nabla \bar{E}-\bar{E} \nabla E)\right)$.

According to (25), we have

$$
\begin{aligned}
\frac{i}{c} \frac{\partial}{\partial t}(E \nabla \bar{E}-\bar{E} \nabla E)= & \frac{1}{2 k_{0}}\left[E \nabla\left(\Delta_{\perp} \bar{E}\right)-\left(\Delta_{\perp} E\right) \nabla \bar{E}+\bar{E} \nabla\left(\Delta_{\perp} E\right)-\left(\Delta_{\perp} \bar{E}\right) \nabla E\right] \\
& +\frac{1}{2} k_{0}\left[E \nabla\left(\left(N_{0}-N\right) \bar{E}\right)+\bar{E} \nabla\left(\left(N_{0}-N\right) E\right)-\left(N_{0}-N\right)(E \nabla \bar{E}+E \nabla \bar{E})\right] \\
& -i \mathbf{K} \cdot \nabla(E \nabla \bar{E}-\bar{E} \nabla E) .
\end{aligned}
$$

The second line of the right hand side reads simply $-k_{0}|E|^{2} \nabla N$, and with classical identities, one can prove that the following relation holds:

$$
E \nabla\left(\Delta_{\perp} \bar{E}\right)-\left(\Delta_{\perp} E\right) \nabla \bar{E}+\bar{E} \nabla\left(\Delta_{\perp} E\right)-\left(\Delta_{\perp} \bar{E}\right) \nabla E=\nabla \cdot \overline{\overline{S_{2}}}
$$


where

$$
\overline{\overline{S_{2}}}=\overline{\overline{1}}\left(E\left(\Delta_{\perp} \bar{E}\right)+\bar{E}\left(\Delta_{\perp} E\right)+2(\nabla E) \cdot(\nabla \bar{E})\right)-(\nabla E)\left(\nabla_{\perp} \bar{E}\right)-\left(\nabla_{\perp} \bar{E}\right)(\nabla E)-\left(\nabla_{\perp} E\right)(\nabla \bar{E})-(\nabla \bar{E})\left(\nabla_{\perp} E\right) .
$$

Thus we get:

$$
\frac{4 k_{0}}{\varepsilon_{0}} \frac{\partial}{\partial t} \mathbf{M}=\frac{1}{2 k_{0}} \nabla \cdot \overline{\overline{S_{2}}}-k_{0}|E|^{2} \nabla N-i \mathbf{K} \cdot \nabla(E \nabla \bar{E}-\bar{E} \nabla E)+\mathbf{K}\left[i\left(\bar{E} \Delta_{\perp} E-E \Delta_{\perp} \bar{E}\right)-2 k_{0} \mathbf{K} \cdot \nabla|E|^{2}\right] .
$$

But, since we have $\nabla \bar{E} \cdot\left(\overline{\overline{1}}-\frac{\mathbf{K K}}{|\mathbf{K}|^{2}}\right) \nabla_{\perp} E=\nabla E \cdot\left(\overline{\overline{1}}-\frac{\mathbf{K K}}{|\mathbf{K}|^{2}}\right) \nabla_{\perp} \bar{E}$, we check that

$$
-i \mathbf{K} . \nabla(E \nabla \bar{E}-\bar{E} \nabla E)+\mathbf{K}\left[i\left(\bar{E} \Delta_{\perp} E-E \Delta_{\perp} \bar{E}\right)=i \nabla \cdot\left[\bar{E} \mathbf{K}\left(\nabla E+\nabla_{\perp} E\right)\right]+\right.\text { c.c. }
$$

In summary, we get

$$
\frac{\partial}{\partial t} \mathbf{M}=\frac{\varepsilon_{0}}{4 k_{0}^{2}} \nabla \cdot \overline{\overline{S_{3}}}-\frac{\varepsilon_{0}}{4}|E|^{2} \nabla N, \quad \text { with } \overline{\overline{S_{3}}}=\frac{1}{2} \overline{\overline{S_{2}}}-2 k_{0}^{2} \mathbf{K K}|E|^{2}+k_{0}\left[i \bar{E} \mathbf{K}\left(\nabla E+\nabla_{\perp} E\right)+\text { c.c. }\right] .
$$

As in Section 2.2, by using (27), we get the global momentum balance relation:

$$
\frac{\partial}{\partial t}\left(\mathbf{M}+m_{I} N_{I} \mathbf{U}\right)+\nabla \cdot\left(m_{I} N_{I} \mathbf{U U}\right)+\nabla\left(P_{e}+P_{I}\right)=\frac{\varepsilon_{0}}{4 k_{0}^{2}} \nabla \cdot \overline{\overline{S_{3}}}-\nabla\left(m_{I} \gamma_{p}|A|^{2} N_{I}\right) .
$$

Notice that, according to (44), we have $\mathbf{K} . \nabla E=O(\epsilon)$, and then, one can show that $\overline{\overline{S_{3}}}=\overline{\overline{S_{1}}}+O(\epsilon)$, where $\overline{\overline{S_{1}}}$ is given by (28).

\subsection{Properties of the advection-Schrödinger equation}

In this subsection, we show, at least in the case where the time derivative is withdrawn, that (46) is a good boundary condition on $\Gamma^{\text {in }}$ for (45), and we give some enlightenments about the well-posedness of the equation. So, after dropping this time derivative, equation (45) reads

$$
i\left(2 \mathbf{K} . \nabla E+E(\nabla . \mathbf{K})+\nu_{0} E\right)+\frac{1}{k_{0}}\left(\Delta_{\perp}^{K} E\right)-k_{0}\left(N-N_{0}\right) E=0 .
$$

For a given function $N$, it is a linear equation. In the case where vector $\mathbf{K}$ is not parallel to the normal vector $\mathbf{n}$ to the boundary $\Gamma^{\text {in }}$, we call it advection-Schrödinger equation and in the special case where $\mathbf{K}$ is parallel to $\mathbf{n}$, it reduces to a classical Schrödinger equation (where the propagation direction plays the part of the time variable).

We give here the main results in the particular case where $\mathcal{D}$ is a half plane whose boundary is $\Gamma^{\mathrm{in}}$.

Lemma. Assume that $\left(k_{0}^{-1} \mathbf{n} . \nabla_{\perp}+2 i \mathbf{n} . \mathbf{K}\right) \alpha^{\text {in }} \in L^{2}(\partial D)$. If $E \in H^{1}(D)$ is a solution to (49), (46).

i) The following energy relation holds

$$
\int_{\mathcal{D}} \nu_{0}|E|^{2} \mathrm{~d} x+\int_{\Gamma^{\text {in }}}|\mathbf{n} \cdot \mathbf{K}||E|^{2} \mathrm{~d} \Gamma(x) \leq-\operatorname{Im}\left(\int_{\Gamma^{\text {in }}} \bar{E}\left(k_{0}^{-1} \mathbf{n} \cdot \nabla_{\perp}+2 i \mathbf{n} \cdot \mathbf{K}\right) \alpha^{\text {in }} \mathrm{d} \Gamma(x)\right) .
$$

ii) Moreover, there exists a constant $C$ depending only on $\mathbf{K}$ such that

$$
\int_{\mathcal{D}} \nu_{0}|E|^{2} \mathrm{~d} x+\int_{\Gamma^{\text {in }}}|\mathbf{n} \cdot \mathbf{K}||E|^{2} \mathrm{~d} \Gamma(x) \leq C \int_{\Gamma^{\text {in }}}\left|\left(k_{0}^{-1} \mathbf{n} \cdot \nabla_{\perp}+2 i \mathbf{n} \cdot \mathbf{K}\right) \alpha^{\mathrm{in}}\right|^{2} \mathrm{~d} \Gamma(x) .
$$


Proof.

i) By multiplying the equation (49) by $\bar{E}$, integrating over $\mathcal{D}$ and taking the complex conjugate, we see that

$$
2 \int_{\mathcal{D}}\left[\nu_{0}|E|^{2}+\nabla \cdot\left(\mathbf{K}|E|^{2}\right)\right] \mathrm{d} x=k_{0}^{-1} \int_{\Gamma^{\mathrm{in}}}\left[-i E\left(\mathbf{n} . \nabla_{\perp} \bar{E}\right)+i \bar{E}\left(\mathbf{n} . \nabla_{\perp} E\right)\right] \mathrm{d} \Gamma(x) ;
$$

but according to (46), we have $i k_{0}^{-1} \mathbf{n} \cdot \nabla_{\perp} E=2 \mathbf{K} . \mathbf{n} E+\left(i k_{0}^{-1} \mathbf{n} \cdot \nabla_{\perp}-2 \mathbf{n} \cdot \mathbf{K}\right)\left(\alpha^{\text {in }}\right)$, then

$$
2 \int_{\mathcal{D}} \nu_{0}|E|^{2} \mathrm{~d} x+\int_{\Gamma^{\text {in }}} 2 \mathbf{n} \cdot \mathbf{K}|E|^{2} \mathrm{~d} \Gamma(x)=\int_{\Gamma^{\text {in }}} 4 \mathbf{n} \cdot \mathbf{K}|E|^{2} \mathrm{~d} \Gamma(x)+\int_{\Gamma^{\text {in }}}\left[i \bar{E}\left(k_{0}^{-1} \mathbf{n} \cdot \nabla_{\perp}+2 i \mathbf{n} \cdot \mathbf{K}\right)\left(\alpha^{\mathrm{in}}\right)+c c\right] \mathrm{d} \Gamma(x),
$$

and we get the result of point i).

ii) According to the previous point, we have

$$
\int_{\mathcal{D}} \nu_{0}|E|^{2} \mathrm{~d} x+|\mathbf{n} \cdot \mathbf{K}| \int_{\Gamma^{\text {in }}}|E|^{2} \mathrm{~d} \Gamma(x) \leq\left(\int_{\Gamma^{\text {in }}}\left|\left(k_{0}^{-1} \mathbf{n} \cdot \nabla_{\perp}+2 i \mathbf{n} \cdot \mathbf{K}\right) \alpha^{\text {in }}\right|^{2} \mathrm{~d} \Gamma(x)\right)^{1 / 2}\left(\int_{\Gamma^{\text {in }}}|E|^{2} \mathrm{~d} \Gamma(x)\right)^{1 / 2}
$$

therefore, we see that

$$
\left(\int_{\Gamma^{\text {in }}}|E|^{2} \mathrm{~d} \Gamma(x)\right)^{1 / 2} \leq \frac{1}{\inf |\mathbf{n} \cdot \mathbf{K}|}\left(\int_{\Gamma^{\text {in }}}\left|\left(k_{0}^{-1} \mathbf{n} \cdot \nabla_{\perp}+2 i \mathbf{n} \cdot \mathbf{K}\right) \alpha^{\mathrm{in}}\right|^{2} \mathrm{~d} \Gamma(x)\right)^{1 / 2},
$$

and plugging this upper-bound into (51), we get the desired result.

If the absorption coefficient $\nu$ is a strictly positive function, the previous result shows that there exists at most one solution $E \in H^{1}(\mathcal{D})$ to (49), (46) for any regular function $\alpha^{\text {in }}$, and the solution is stable with respect to the boundary condition.

To be sure that problem (49), (46) has a solution and is well posed, we now proceed to the analysis after making some new assumptions, for the sake of simplicity. Assume first that $d=2$ and that $\mathcal{D}$ is the half space $\mathcal{D}=\left\{x=\left(x_{1}, x_{2}\right) \in \mathbf{R}^{2} \quad \mid x_{1}>0\right\}$ whose boundary $\Gamma^{\text {in }}$ corresponds to $x_{1}=0$. Assume also that $N-N_{0}=0$, that $\mathbf{K}=\left(K_{1}, K_{2}\right)$ is constant and that the coefficient $\nu_{0}>0$ is constant. Then relations (49), (46) may read as

$$
\begin{aligned}
& i\left(K_{1} \partial_{x_{1}}+K_{2} \partial_{x_{2}}\right) E+\frac{1}{2} k_{0}^{-1}\left(K_{2}^{2} \partial_{x_{1} x_{1}}-\right.\left.2 K_{1} K_{2} \partial_{x_{1} x_{2}}+K_{1}^{2} \partial_{x_{2} x_{2}}\right) E+i \nu_{0} E=0, \\
& \frac{i k_{0}^{-1}}{2|\mathbf{K}|}\left(K_{1} K_{2} \partial_{x_{2}}-K_{2}^{2} \partial_{x_{1}}\right) E+K_{1} E=K_{1} g \quad \text { on } \Gamma^{\text {in }},
\end{aligned}
$$

where $g=\left(1+\frac{i k_{0}^{-1}}{2 K_{1}|\mathbf{K}|}\left(K_{1} K_{2} \partial_{x_{2}}-K_{2}^{2} \partial_{x_{1}}\right)\right) \alpha^{\text {in }}$.

Let us denote $\widehat{\bullet}$ the Fourier transform associated to $x_{2}$ (with $\xi$ the Fourier variable) and $\sqrt{ }$. the principal determination of the square root. Then, if we set

$$
R_{-}(\xi)=i \frac{K_{1}}{K_{2}} \xi-i \frac{K_{1}}{K_{2}^{2}}|\mathbf{K}|\left(1-\sqrt{1-2 \frac{|\mathbf{K}|}{k_{0}} \frac{K_{2}}{K_{1}^{2}} \xi+2 i \frac{\nu_{0}}{k_{0}} \frac{K_{2}^{2}}{K_{1}^{2}}}\right)
$$

we have the following result ( $\mathcal{S}^{\prime}$ denotes the space of tempered distributions).

Proposition. Let $g \in \mathcal{S}^{\prime}\left(\mathbf{R}_{x_{2}}\right)$. Problem (52), (53) has a unique solution $E$ in $\mathcal{S}^{\prime}(\mathcal{D})$; it is given by:

$$
\widehat{E}\left(x_{1}, \xi\right)=\widehat{g}(\xi) \mathrm{e}^{R_{-}(\xi) x_{1}}\left(1-\frac{1}{2 k_{0}}+\frac{1}{2 k_{0}} \sqrt{1-2 \frac{|\mathbf{K}|}{k_{0}} \frac{K_{2}}{K_{1}^{2}} \xi+2 i \frac{\nu_{0}}{k_{0}} \frac{K_{2}^{2}}{K_{1}^{2}}}\right)^{-1} .
$$




\section{Sketch of the proof.}

We set $\nu=\nu_{0}|\mathbf{K}|^{-1}, \eta=k_{0}^{-1}|\mathbf{K}|, k_{j}=K_{j}|\mathbf{K}|^{-1}$, and define $P(X, Y)$ as the polynomial which characterizes the differential operator of equation (52):

$$
P\left(\partial_{x_{1}}, \partial_{x_{2}}\right)=i\left(k_{1} \partial_{x_{1}}+k_{2} \partial_{x_{2}}\right)+\frac{1}{2} \eta\left(k_{2}^{2} \partial_{x_{1}}^{2}-2 k_{1} k_{2} \partial_{x_{1}} \partial_{x_{2}}+k_{1}^{2} \partial_{x_{2}}^{2}\right)+i \nu .
$$

If we write $E_{0}\left(x_{2}\right)=E\left(0, x_{2}\right)$, the Fourier transform of the equation reads:

$$
P\left(\partial_{x_{1}}, i \xi\right) \widehat{u}\left(x_{1}, \xi\right)=\frac{\eta k_{2}^{2}}{2}\left[\left(\frac{1}{\eta} \frac{2 i k_{1}}{k_{2}^{2}} \widehat{g}(\xi)-i \frac{k_{1}}{k_{2}} \xi \widehat{E_{0}}(\xi)\right) \delta_{x=0}+\widehat{E_{0}}(\xi) \delta_{x=0}^{\prime}\right] .
$$

But $P\left(\partial_{x_{1}}, i \xi\right)$ considered as a polynomial with respect to $\partial_{x_{1}}$ may be recasted as follows:

$$
P\left(\partial_{x_{1}}, i \xi\right)=\frac{k_{0}^{-1} k_{2}^{2}}{2}\left(\partial_{x_{1}}-R_{+}(\xi)\right)\left(\partial_{x_{1}}-R_{-}(\xi)\right),
$$

where

$$
R_{ \pm}(\xi)=i \frac{k_{1}}{k_{2}} \xi-i \frac{k_{1}}{k_{2}^{2}}\left(1 \pm \sqrt{1-2 \frac{\eta k_{2}}{k_{1}^{2}} \xi+2 i \nu \frac{\eta k_{2}^{2}}{k_{1}^{2}}}\right) .
$$

Thus, a formal calculus shows that $\widehat{u}\left(x_{1}, \xi\right)$ has to be of the following form:

$$
\widehat{E}\left(x_{1}, \xi\right)=B_{-}(\xi) \mathrm{e}^{R_{-}(\xi) x_{1}}+B_{+}(\xi) \mathrm{e}^{R+(\xi) x_{1}} .
$$

Since $\nu>0$, one can see that $\operatorname{Re}\left(\mathrm{R}_{+}(\xi)\right)>0, \operatorname{Re}\left(R_{-}(\xi)\right)<0$. So, in order to have a bounded function when $x_{1}$ goes to infinity, it is necessary that $B_{+}(\xi)=0$. Then we get

$$
\widehat{E}\left(x_{1}, \xi\right)=\widehat{E}(0, \xi) \mathrm{e}^{R_{-}(\xi) x_{1}} .
$$

To get the desired result, it is enough to notice that, according to (53), we must have

$$
\widehat{E}(0)+\frac{i}{2 k_{0} K_{1}|\mathbf{K}|}\left(i K_{1} K_{2} \xi-K_{2}^{2} R_{-}(\xi)\right) \widehat{E}(0)=\widehat{g} .
$$

According to this proposition, we see that for $x_{1}>0$, we get

$$
\left(\partial_{x_{1}}-R_{-}\left(\xi_{2}\right)\right) \widehat{u}\left(x_{1}, \xi_{2}\right)=0 .
$$

This property may be interpreted as follows: equation (52), which is of second order with respect to the derivative $\partial_{x_{1}}$, may be replaced by an equation of first order with respect to the derivative $\partial_{x_{1}}$.

The proposition implies that if $g \in H_{x_{2}}^{1 / 2}$, then $E \in H^{1}(\mathcal{D})$, and there exists a unique solution of (49) in the space $H^{1}(\mathcal{D})$. Equation (49) is deeply studied in [20] in the case where $\mathbf{K}$ is constant and $\mathcal{D}$ is a half plane or a parallelepiped. For a numerical treatment of (49), we refer to [21].

Remark (extension). If there are two laser beams entering in the simulation domain which are characterized by the propagation vectors $\mathbf{e}_{b}^{1}$ and $\mathbf{e}_{b}^{2}$, then one can define two solutions of the eikonal equations.

$$
\begin{array}{ll}
\left|\nabla \phi^{1}\right|^{2}=1-N,\left.\quad \nabla \phi^{1}\right|_{\Gamma^{\text {in }}}=\mathbf{e}_{b}^{1}\left(1-N_{0}\right)^{1 / 2}, \\
\left|\nabla \phi^{2}\right|^{2}=1-N, & \left.\nabla \phi^{2}\right|_{\Gamma^{\text {in }}}=\mathbf{e}_{b}^{2}\left(1-N_{0}\right)^{1 / 2}
\end{array}
$$


and we set $\mathbf{K}^{1}=\nabla \phi^{1}, \mathbf{K}^{2}=\nabla \phi^{2}$. Then the laser fields $E^{1}, E^{2}$ solve

$$
i\left(2 \mathbf{K}^{h} . \nabla E^{h}+E^{h}\left(\nabla \cdot \mathbf{K}^{h}\right)+\nu_{0} E^{h}\right)+\frac{1}{k_{0}}\left(\Delta_{\perp}^{h} E^{h}\right)-k_{0}\left(N-N_{0}\right) E^{h}=0,
$$

supplemented with the good boundary conditions with the incoming laser fields $a^{i n, 1}$ and $a^{i n, 2}$.

These equations are coupled with the hydrodynamic ones through the modified momentum equation obtained by writing in the right hand side of (47) the term $\nabla\left(\left|E^{1}\right|^{2}+\left|E^{2}\right|^{2}\right)$ instead of $\nabla\left(|E|^{2}\right)$.

\subsection{Particular case: the classical paraxial equation}

The laser beam is now assumed to enter in the plasma without any incidence angle, i.e., the unit vector $\mathbf{e}_{b}$ is equal to the inwards normal vector $-\mathbf{n}$ to the boundary $\Gamma^{\text {in }}$. Denote by $z$ the space variable in the direction $\mathbf{e}_{b}: z=\mathbf{e}_{b} . x$ and $x_{\perp}$ the transverse variable. Moreover, assume that $N_{0}$ depends only on the $z$ variable; then the solution of the eikonal equation is trivial:

$$
\mathbf{K}=\mathbf{e}_{b} K(z), \quad K(z)=\sqrt{1-N_{0}(z)}
$$

and equations (45), (46) read as

$$
i\left(\frac{2}{c} \frac{\partial E}{\partial t}+2 K \frac{\partial E}{\partial z}+E \frac{\partial K}{\partial z}+\nu_{0} E\right)+\frac{1}{k_{0}}\left(\Delta_{\perp} E\right)-k_{0}\left(N-N_{0}\right) E=0,
$$

supplemented with an initial condition and a boundary condition

$$
E(t, .)=\alpha^{\text {in }}(t, .), \quad \text { on } \Gamma^{\text {in }} .
$$

This equation is very close to equation (2) of [9]; in that reference, there is an extra term corresponding to a time derivative of the phase $\phi$ and a corrector to the transverse Laplace operator (which has been introduced with a poor justification in [24] for "non paraxial propagation").

Assume for a while that the model is posed in the half space $\{z \geq 0\}$. By multiplying equation (56) by $\bar{E}$, integrating with respect to the transverse directions and taking the complex conjugate, we see that

$$
\frac{1}{c} \frac{\partial}{\partial t}\left(\int_{\mathbf{R}^{2}}|E|^{2} \mathrm{~d} x_{\perp}\right)+\frac{\partial}{\partial z}\left(\int_{\mathbf{R}^{2}} K|E|^{2} \mathrm{~d} x_{\perp}\right)+2 \int_{\mathbf{R}^{2}} \nu_{0}|E|^{2} \mathrm{~d} x_{\perp}=0 .
$$

Thus, if $\alpha$ does not depend on $t$ and if a stationary solution is reached, then this solution satisfies, for any $z$ positive,

$$
K(z) \int_{\mathbf{R}^{2}}\left|E\left(z, x_{\perp}\right)\right|^{2} \mathrm{~d} x_{\perp}+2 \int_{0}^{z} \int_{\mathbf{R}^{2}} \nu_{0}\left|E\left(z^{\prime}, x_{\perp}\right)\right|^{2} \mathrm{~d} x_{\perp} \mathrm{d} z^{\prime}=K(0) \int_{\mathbf{R}^{2}}\left|\alpha\left(x_{\perp}\right)\right|^{2} \mathrm{~d} x_{\perp} ;
$$

that is to say if there is no absorption, then the flux of the laser energy $K \int_{\mathbf{R}^{2}}\left|E\left(., x_{\perp}\right)\right|^{2} \mathrm{~d} x_{\perp}$ is conserved when the laser propagates.

\subsection{Numerics for the classical paraxial equation}

For the simulation of laser propagation in Inertial Confinement Fusion plasma, one must often deal numerically with the hydrodynamic system (26), (27) coupled with the equation of propagation (56) on a spatial domain $\mathcal{D}$ whose sizes are typically of the order of one millimeter (the vector $\mathbf{e}_{b}$ is parallel to an edge of the box).

In order to have a good discretization of the speckles, the mesh size in the transverse directions has to be of the order of a fraction of a micron, the mesh size $\delta z$ in the $z$ direction may be 2 or 3 times larger. Denote by $\delta t$ the time step. For the hydrodynamic system, one has to use a time step satisfying the CFL condition of the 
type $\max \left(|\mathbf{U}|, c_{s}\right) \delta t / \min (\delta x, \delta y, \delta z)<1$. Therefore we expect to use a numerical method which is stable with time step such that $c_{s} \delta t / \delta z$ be in the order of 1 , then $c \delta t / \delta z$ will be very large compared to 1 .

For numerical purpose, the simulation domain is generally a parallelepiped where one edge is parallel to $z$, and one must state boundary conditions on transverse boundaries $\Gamma_{\perp}$ : it may be seen that Neuman condition corresponds to a reflection of the light on $\Gamma_{\perp}$ which not well suited. When dealing with this problem, one can withdraw the time derivative and the absorption; the behavior of the solution corresponds to a solution of a Schrödinger equation $\frac{\partial E}{\partial z}-i \frac{1}{2 k_{0} K} \Delta_{\perp} E$. From a theoretical point of view, it is a difficult problem to find good transparent boundary conditions for this equation (these conditions involve pseudo-differential operators on $\Gamma_{\perp}$, see [2] for instance); see below for a numerical treatement.

\subsubsection{Numerical scheme}

We consider a Cartesian mesh of finite difference type. At each time step $\left[t^{n-1}, t^{n-1}+\delta t\right]$, one has to solve successively the hydrodynamic system (26), (27) and the paraxial equation of propagation (56). For the hydrodynamics system, an explicit numerical scheme of Lagrange-Euler type may be used for example: in the Lagrange step, one uses a classical Godunov scheme to evaluate intermediate values of the density and the velocity, the ponderomotive force $\gamma_{p} \nabla|E|^{2}$ is taken into account by a standard centered discretization; afterwards, a second order projection technique with VanLeer slope limiters is used for the advection part (see [28] for details).

Let us focus now on the treatment of the paraxial equation (56) in a 2D geometry (for the sake of simplicity). Let $y$ be the transverse direction and denote by $E_{j, m}^{n}$ the evaluation of $E$ at time $t^{n}=n . \delta t$ at position $z_{j}=j . \delta z$ and $y_{m}=m . \delta y$.

If there was only a propagation phenomenon, that is to say if one had to deal with the advection operator $\left(\frac{1}{c} \frac{\partial}{\partial t}+K \frac{\partial}{\partial z}+\nu_{0}\right)$, we would use a time implicit scheme and we would get the values $E_{j}^{n}$ at time $t^{n}$ from step to step in the direction of propagation from the entrance side $z=0$. Here, we perform a splitting of the equation (56), with respect to the $z$ variable. We move from $E_{j, \cdot}^{n}$ to $E_{j+1, \cdot}^{n}$. through the intermediate state $E_{j+1, \cdot}^{n}$. i) The first step: advection. The values $E_{j \text {, }}^{n}$ are assumed to be known and we have to solve between $z_{j}$ and $z_{j+1}$ the advection equation:

$$
\frac{1}{c} \frac{\partial E}{\partial t}+K \frac{\partial E}{\partial z}+\left(\nu_{0}+\frac{1}{2} \frac{\partial K}{\partial z}\right) E=0
$$

Let us denote $\mu_{j+1 / 2}=\frac{1}{2}\left(\nu_{0, j}+\nu_{0, j+1}\right)+\left(K_{j+1}-K_{j}\right) / 2 \delta z$. One could get $\widetilde{E_{j+1}^{n} \text {, }}$ by a classical upwind scheme with respect to the $z$ variable:

$$
\frac{1}{c} \frac{E_{j, m}^{n}-E_{j, m}^{n-1}}{\delta t}+K_{j} \frac{\widetilde{E_{j+1, m}^{n}}-E_{j, m}^{n}}{\delta z}+\frac{\mu_{j+1 / 2}}{4}\left(E_{j, m}^{n}+\widetilde{E_{j+1, m}^{n}}\right)=0 .
$$

This method is refered below as the naive method. We now propose an improved method to deal with the advection equation: we do not solve it directly, but the corresponding equation for the square of the modulus of $E$, that is to say $\left(\frac{1}{c} \frac{\partial}{\partial t}+K \frac{\partial}{\partial z}\right)|E|^{2}+\left(2 \nu_{0}+\frac{\partial K}{\partial z}\right)|E|^{2}=0$, which yields

$$
\left.\widehat{\mid E_{j+1, m}^{n}}\right|^{2}=\left(\left|E_{j, m}^{n}\right|^{2}\left(1-\eta-\frac{\mu_{j+1 / 2} \delta z}{2 K_{j}}\right)+\eta\left|E_{j, m}^{n-1}\right|^{2}\right)\left(1+\frac{\mu_{j+1 / 2} \delta z}{2 K_{j}}\right)^{-1}, \quad \text { with } \eta=\frac{1}{c K} \frac{\delta z}{\delta t},
$$

and afterwards a simple advection equation for the quantity of $E_{.}^{n} /\left|E_{.}^{n}\right|$ that is related to the argument of $E_{.}^{n}$

ii) The second step: diffraction. Denote now $\delta N=N-N_{0}$. Once $\widetilde{E_{j+1, *}^{n}}$ is obtained, there are two ways to find $E_{j+1, *}^{n}$ by solving

$$
K \frac{\partial E}{\partial z}=\frac{i}{2 k_{0}}\left(\Delta_{\perp} E\right)-i \frac{k_{0}}{2}(\delta N) E
$$


In the first way, we discretize the Laplace operator $\Delta_{\perp}$ by a Crank-Nicolson technique:

$$
K \frac{E_{j+1, m}^{n}-\widetilde{E_{j+1, m}^{n}}}{\delta z}=\frac{i}{2 k_{0}}\left(\left(\Delta_{\perp} \widetilde{E_{j+1}^{n}}\right)_{m}+\left(\Delta_{\perp} E_{j+1}^{n}\right)_{m}\right)-i \frac{k_{0}}{2}(\delta N)_{j+1 / 2}\left(\widetilde{E_{j+1, m}^{n}}+E_{j+1, m}^{n}\right),
$$

where $\left(\Delta_{\perp} E\right)_{m}$ stands here for the discretized form of the transverse Laplace operator $\left(2 E_{m}-E_{m+1}-\right.$ $\left.E_{m-1}\right) \delta y^{-2}$. To deal with the transparent boundary conditions on $\Gamma_{\perp}$ from a numerical point of view, the so called Perfectly Matched Layers (PML) approach leads to a very efficient method which has been proposed for Schrödinger equation in [17]; this method has also been implemented in the numerical code HERA ( $c f$. [3]), with which the following numerical results are obtained.

In the second way, one uses an analytic formula to deal with the $i \frac{k_{0}}{2} \delta N$ term and a spectral method for transverse Laplace operator. More precisely, if $\mathcal{F}\left(E_{j}\right)$ denotes the value of the Fourier Transform in the transverse direction of the function $E_{j}(y)$ and $\xi$ the corresponding Fourier variable, we simply set

$$
\mathcal{F}\left(E_{j+1}\right)(\xi)=\exp \left(-i \frac{\xi^{2}}{2 K k_{0}} \delta z\right) \mathcal{F}\left(\widetilde{E_{j+1}^{n}}\right)(\xi)
$$

and

$$
E_{j+1}^{n}=\exp \left(i \frac{k_{0}}{2 K}(\delta N)_{j+1 / 2} \delta z\right) \mathcal{F}^{-1}\left(\mathcal{F}\left(E_{j+1}\right)\right) .
$$

Of course, from a numerical point of view, the Fast Fourier Transform (FFT) and the inverse FFT are used for $\mathcal{F}$ and $\mathcal{F}^{-1}$. To deal with non reflective boundary conditions on $\Gamma_{\perp}$, it is necessary to add some ad hoc artificial absorbing coefficient in boundary layers.

\subsubsection{Properties of the numerical schemes}

The natural criterium for such an implicit scheme is

$$
\sup \frac{\delta z}{c K_{j} \delta t}<1
$$

If one assumes that $K$ is constant, one can see that (58) yields:

$$
\left.\widetilde{\mid E_{j+1, m}^{n}}\right|^{2} \leq \max \left(\left|E_{j, m}^{n}\right|^{2},\left|E_{j, m}^{n-1}\right|^{2}\right),
$$

and in the general case, one can check that

$$
K_{j+1}\left|\widetilde{E_{j+1, m}^{n}}\right|^{2} \leq K_{j} \max \left(\left|E_{j, m}^{n}\right|^{2},\left|E_{j, m}^{n-1}\right|^{2}\right) .
$$

For the sake of simplicity, assume that there are reflection boundary conditions (that is to say Neuman condition) on the boundary in the transverse direction; then for the Crank-Nicolson technique, according to (59), we get:

$$
\begin{aligned}
& \sum_{m} K \frac{E_{j+1, m}^{n}-\widetilde{E_{j+1, m}^{n}}}{\delta z}\left(\overline{E_{j+1, m}^{n}}+\widetilde{E_{j+1, m}^{n}}\right)+c . c . \\
& \quad \sum_{m}\left(-\frac{i}{4}(\delta N)_{j+1 / 2, m}\left(\widetilde{E_{j+1, m}^{n}}+E_{j+1, m}^{n}\right)+\frac{i}{2 k_{0}}\left(\left(\Delta_{\perp} \widetilde{E_{j+1}^{n}}\right)_{m}+\left(\Delta_{\perp} E_{j+1}^{n}\right)_{m}\right)\right)\left(\overline{E_{j+1, m}^{n}}+\widetilde{\widetilde{E_{j+1, m}^{n}}}\right)+c . c .
\end{aligned}
$$

By applying the standard relation $\Sigma_{m}\left(2 u_{m}-u_{m+1}-u_{m-1}\right) \overline{u_{m}}=\Sigma_{m}\left(u_{m}-u_{m+1}\right)\left(\overline{u_{m}}-\overline{u_{m+1}}\right) \in \mathbf{R}$ to $u=$ $\widetilde{E_{m+1}^{n}}+E_{m+1}^{n}$, we easily check that the right hand side of the above equation is zero (this is the discrete 


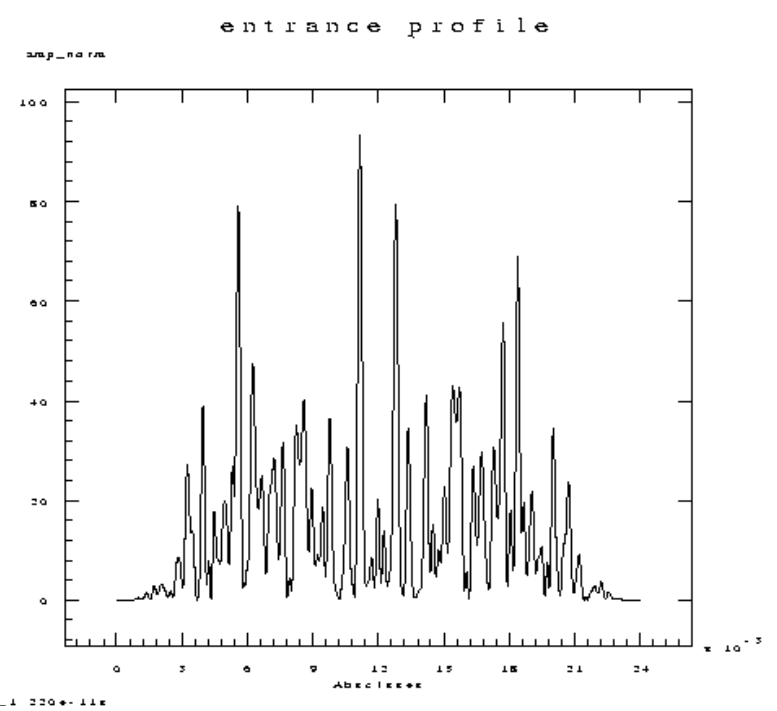

FiguRE 1. Incoming boundary condition. The laser intensity versus the transverse space variable.

counterpart of the relation $\left.\int_{\mathbf{R}} \overline{E(y)} \Delta_{y} E(y) \mathrm{d} y \in \mathbf{R}\right)$. Then, if we denote by $\left\|E_{j}\right\|$ the $l^{2}$ norm in the transverse direction $\left(\left\|E_{j}\right\|^{2}=\sum_{m}\left|E_{j, m}\right|^{2}\right)$ we have the conservation of energy during the second step:

$$
\left\|E_{j+1}^{n}\right\|^{2}=\left\|\widetilde{E_{j+1}^{n}}\right\|^{2}
$$

Therefore, (60) yields:

and, by induction, we see that

$$
\left\|E_{j+1}^{n}\right\|^{2} \leq \max \left(\left\|E_{j}^{n}\right\|^{2},\left\|E_{j}^{n-1}\right\|^{2}\right)
$$

$$
\sup _{j}\left\|E_{j}^{n}\right\|^{2} \leq \max \left(\sup _{N}\left(\left\|\alpha^{i n, N}\right\|^{2}\right), \sup _{j}\left\|E_{j}^{0}\right\|^{2}\right), \quad \forall n .
$$

This expresses that the proposed splitting scheme is stable for the norm $\sup _{j} K_{j}\left\|E_{j}\right\|^{2}$, see [3] for the details.

\subsubsection{Numerical illustration}

One considers here a $2 \mathrm{D}$ problem in a simulation box whose dimensions are

$$
L_{z, \max }=560 \mu \mathrm{m}, \quad L_{y, \max }=240 \mu \mathrm{m} \text {. }
$$

The incoming boundary condition in $z=0$, corresponds to a model of a laser beam whose width is equal to $200 \mu \mathrm{m}$, in which there are a lot of speckles (i.e., hot spots). It may be seen in Figure 1 that it is a highly randomly oscillating function. The width of the speckles at the incoming boundary is about 3 microns.

In the framework of the model (26), (27), we choose the sound speed in the form

$$
c_{s}^{2}=\left(T_{e}+3 T_{I}^{i n i} \frac{N^{2}}{N^{i n i^{2}}}\right) \frac{k_{B}}{m_{I}}
$$

where $k_{B}$ is the Boltzmann constant, the electron temperature $T_{e}$ is equal to $30.10^{6}$ Kelvin, the initial ion temperature $T_{I}^{i n i}$ is equal to $3 \times 10^{6}$ Kelvin and the initial density is equal to $N^{i n i}=0.1$. 


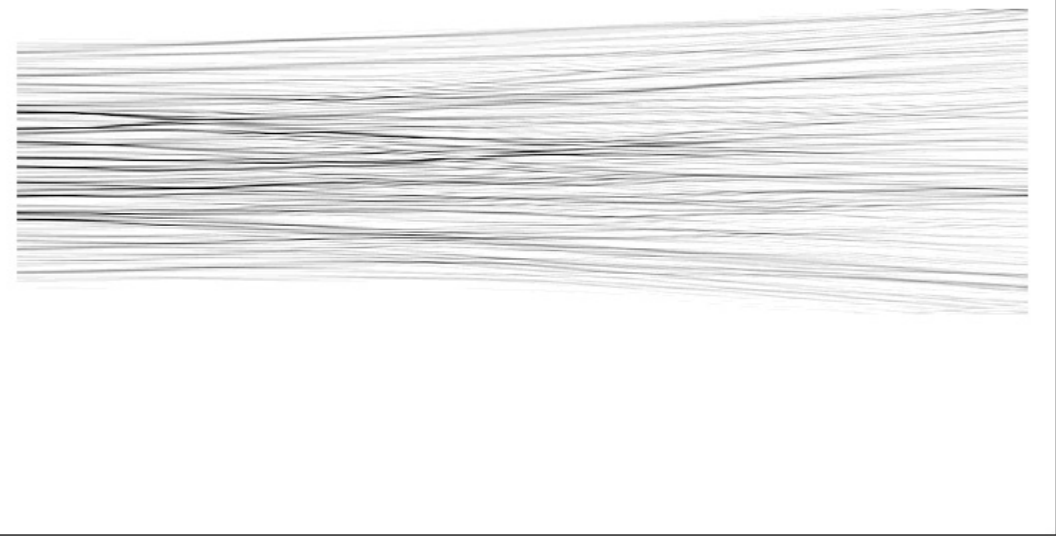

FiguRE 2. Map of the laser intensity (with a greyscale map) with the naive numerical method. The laser comes from the left hand side. The integral of the outgoing intensity over the transverse direction is much smaller than the integral of the incoming intensity.

The space step in the transverse directions is $\delta y=0.24 \mu \mathrm{m}$ and 3 times larger in the $z$ direction. The sound speed is about $7 \times 10^{7} \mathrm{~cm} / \mathrm{s}$ and the velocity of the matter is 10 times smaller. Then, according to the classical CFL condition, the time step is about $0.3 \mathrm{ps}$; so $K c \delta t / \delta z$ is in the order of 125 . There are about 800000 cells. We always use here the Crank-Nicolson method for dealing with the diffraction. It is necessary to wait at least $10 \mathrm{ps}$ in order to have an adequate response of the plasma. We look at the laser intensity at the time $12 \mathrm{ps}$. We compare the results obtained with the naive method of discretization for the advection step (based on (57)) and the one obtained with the improved method (based on (58)). One can see in Figure 2 the map of the laser intensity with both methods. It is clear that the results are better with the improved method indeed the global laser energy outgoing from the rear side equals the one incoming in the domain (up to the energy which goes away through the PML Layers).

\section{ON THE MODELLING OF BRILlOUIN INSTABILITY}

We are looking here at an instability which occurs by the coupling of three waves: an ion acoustic wave (which is a perturbation of ion density), the main laser wave which travels forwards (called pump wave) and a backscattered laser wave which travels backwards (called stimulated Brillouin backscattered wave). Let us assume that the density $N$ is close to a constant $N_{\text {ref }}$ (which plays the part of $N_{0}$ ) and that the vector $\mathbf{e}_{b}$ characterizing the direction of the pump wave equals to $-\mathbf{n}$, then wave vector $\mathbf{K}$ is equal to $\sqrt{1-N_{r e f}} \mathbf{e}_{b}$. After defining

$$
k_{p}=k_{0} \sqrt{1-N_{\text {ref }}}
$$

the key point in the sequel is to assume that the fields $A$ may be decomposed into two components:

$$
A=E_{p} \mathrm{e}^{i k_{p} z}+E_{m} \mathrm{e}^{-i k_{p} z}
$$

where the functions $E_{p}, E_{m}$ are weakly space varying and correspond to the pump wave and the backscattered wave respectively. In the sequel, we first explain how to switch from the model where the laser field satisfies (25) 
Figure 3. Map of the laser intensity (with the same greyscale map as in Fig. 2). Simulation with the improved numerical method with the same numerical conditions. The integral of the outgoing intensity over the transverse direction is about the same than the integral of the incoming intensity.

and the plasma hydrodynamics is described by (26), (27), to model which, besides the macroscopic hydrodynamics system, consists in a paraxial equation for the main laser wave, a paraxial equation for the back-scattered laser wave and an evolution equation for the ion acoustic wave. Secondly, we focus on simplifications of this system in order to obtain the so called standard decay system (it corresponds to a three-wave coupling system). Afterwards, we shall analyse the three-wave coupling system and propose numerical techniques to deal with it.

\subsection{First Brillouin model}

Let us define $\beta=c k_{0} N_{\text {ref }} / 2$ and $c_{g}=c \sqrt{1-N_{\text {ref }}}$ the group velocity. By using these notations, if there was no back-scattered wave $E_{m}$, the classical paraxial equation (56) would read

$$
\frac{\partial E_{p}}{\partial t}+c_{g} \frac{\partial E_{p}}{\partial z}+\nu_{a} E_{p}-i \frac{c}{2 k_{0}} \Delta_{\perp} E_{p}+i \beta\left(\frac{N}{N_{r e f}}-1\right) E_{p}=0
$$

where the absorption coefficient is given by $\nu_{a}=\frac{1}{2} c \nu_{0}$.

Let us denote

$$
k_{s}=2 k_{p} .
$$

Then, after neglecting $\nabla E_{p, m}$ with respect to $i k_{p} E_{p, m}$, we get:

$$
\nabla|A|^{2} \simeq \nabla\left(\left|E_{p}\right|^{2}+\left|E_{m}\right|^{2}\right)+\mathbf{e}_{b}\left[i k_{s} E_{p} \overline{E_{m}} \mathrm{e}^{i k_{s} z}+\text { c.c. }\right]
$$

Thus, we see that a plasma wave with a wave number $k_{s}$ may be excited. So, we decompose the plasma flow $N, \mathbf{U}$ into basic components $N^{0}, \mathbf{U}^{0}$ (which are slowly varying functions on the space variable) and a perturbation wave characterized by $N^{1}, \mathbf{U}^{1}$ which are highly oscillating functions with respect to $z$ according to the frequency $k_{s}$. We set:

$$
N \simeq N^{0}+N^{1}, \quad \mathbf{U} \simeq \mathbf{U}^{0}+\mathbf{U}^{1}
$$


The perturbation wave is called the ion acoustic wave associated to Brillouin instability. We have to decompose the initial system into two sub-systems. The first one is the zero order system which is called basic hydrodynamics system:

$$
\begin{aligned}
& \frac{\partial}{\partial t} N^{0}+\nabla\left(N^{0} \mathbf{U}^{0}\right)=0, \\
& \frac{\partial}{\partial t}\left(N^{0} \mathbf{U}^{0}\right)+\nabla\left(N^{0} \mathbf{U}^{0} \mathbf{U}^{0}\right)+c_{s}^{2} \nabla N^{0}=-\gamma_{p} N^{0} \nabla\left(\left|E_{p}\right|^{2}+\left|E_{m}\right|^{2}\right) .
\end{aligned}
$$

The second one is obtained by combining (26)-(27), (61)-(62) and neglecting the terms like $\nabla\left(N^{1} \mathbf{U}^{1}\right)$, $N^{1} \mathbf{U}^{0} . \nabla \mathbf{U}^{1}, N^{1} \mathbf{U}^{1} . \nabla \mathbf{U}^{0}$, that is to say

$$
\begin{aligned}
\frac{\partial}{\partial t} \frac{N^{1}}{N^{0}}+\mathbf{U}^{0} \cdot \nabla \frac{N^{1}}{N^{0}}+\nabla \cdot \mathbf{U}^{1}+\mathbf{U}^{1} \cdot \nabla \log N^{0} & =0 \\
N^{0}\left(\frac{\partial}{\partial t} \mathbf{U}^{1}+\mathbf{U}^{1} \cdot \nabla \mathbf{U}^{0}+\mathbf{U}^{0} \cdot \nabla \mathbf{U}^{1}\right)+c_{s}^{2} N^{0} \nabla \frac{N^{1}}{N^{0}} & =-\gamma_{p} N^{0} \nabla\left(|A|^{2}-\left|E_{p}\right|^{2}-\left|E_{m}\right|^{2}\right) .
\end{aligned}
$$

We now set

$$
N^{1}=N^{0}\left[n \mathrm{e}^{i k_{s} z}+\text { c.c. }\right], \quad \mathbf{U}^{1}=\mathbf{v e}^{i k_{s} z}+\text { c.c. }
$$

Then we get:

$$
\begin{gathered}
\partial_{t} n+\mathbf{U}^{0} \cdot \nabla n+i k_{s} \mathbf{U}^{0} \cdot \mathbf{e}_{b} n+i k_{s} \mathbf{v} \cdot \mathbf{e}_{b}+\nabla \cdot \mathbf{v}=-\mathbf{v} \cdot \nabla \log \left(N^{0}\right), \\
\partial_{t} \mathbf{v}+\mathbf{U}^{0} \cdot \nabla \mathbf{v}+\left(i k_{s} \mathbf{U}^{0} \cdot \mathbf{e}_{b}\right) \mathbf{v}+c_{s}^{2} i k_{s} \mathbf{e}_{b} n+c_{s}^{2} \nabla n=-\mathbf{v} \cdot \nabla \mathbf{U}^{0}-i \gamma_{p} k_{s} \mathbf{e}_{b} E_{p} \overline{E_{m}} .
\end{gathered}
$$

According to the same paraxial approximation as before, if we take the contribution of $A N^{1}$ corresponding to $\mathrm{e}^{i k_{0} z}$ and $\mathrm{e}^{-i k_{0} z}$, we get the following propagation equations for the two laser waves:

$$
\begin{aligned}
\partial_{t} E_{p}+c_{g} \partial_{z} E_{p}+\nu_{a} E_{p}-i \frac{c}{2 k_{0}} \Delta_{\perp} E_{p} & =i \beta(1-M) E_{p}-i \beta M n E_{m}, \\
\partial_{t} E_{m}-c_{g} \partial_{z} E_{m}+\nu_{a} E_{m}-i \frac{c}{2 k_{0}} \Delta_{\perp} E_{m} & =i \beta(1-M) E_{m}-i \beta M \bar{n} E_{p}
\end{aligned}
$$

with

$$
M=\frac{N^{0}}{N_{r e f}} .
$$

The system of equations (63)-(66) is a first model for the Brillouin instability, knowing that the main flow $\left(N^{0}, \mathbf{U}^{0}\right)$ is given. A strong coupling occurs in the right hand side of the previous equations (which will lead to numerical difficulties). In the sequel we will change the previous model for a simpler one.

Remark. Since we have neglected the terms like $\nabla\left(N^{1} \mathbf{U}^{1}\right)$, we cannot hope that this last system (63), (64) coupled with basic hydrodynamics ones yields some momentum balance equation. If we got such a conservation property, it would be necessary to put in (61), (62) the contributions "at zero order" of the neglected terms that is to say to add the term $-\nabla\left(N^{0}(n \overline{\mathbf{v}}+\bar{n} \mathbf{v})\right)$ at the right hand side of (61) and the term $-N^{0} i k_{s} \mathbf{U}^{0}$. $(n \overline{\mathbf{v}}+$ $\bar{n} \mathbf{v})-N^{0}(n \overline{\mathbf{v}}+\bar{n} \mathbf{v}) \cdot \nabla \mathbf{U}^{0}$ at the right hand side of (62). For details concerning this remark, see [34, 39].

\subsection{Reduction to a three-wave coupling model}

We do not take into account here the transverse phenomena: all the quantities are assumed to depend on $t$ and $z$ only. We first show that when $E_{p}$ is equal to a fixed value, this linear system which consists in (63), (64) and (66) exhibits an excited mode which may be also produced by a simpler one which consists only in two equations; we will re-introduce the equation describing the evolution of $E_{p}$ afterwards (for a physical explanation of this reduction, see [38]). 
Denote by $U=\mathbf{U}^{0} \cdot \mathbf{e}_{b}$ the longitudinal component of $\mathbf{U}^{0}$, and

$$
w=c_{s}^{-1} \mathbf{v} \cdot \mathbf{e}_{b},
$$

the dimensionless component of $\mathbf{v}$. Then equations (63), (64) read as

$$
\begin{aligned}
& \partial_{t} n+U \partial_{z} n+i k_{s} U n+i k_{s} c_{s} w+c_{s} \partial_{z} w=-c_{s} w \partial_{z}(\log M) \\
& \partial_{t} w+U \partial_{z} w+i k_{s} U w+i k_{s} c_{s} n+c_{s} \partial_{z} n=-i \gamma_{p} k_{s} c_{s}^{-1} E_{p} \overline{E_{m}}-w \partial_{z} U
\end{aligned}
$$

For the sake of simplicity, we also assume for a while that

$$
\nu_{a}=0, \quad M=1, \quad U \text { is constant. }
$$

The evolution system for $n, w, E_{m}$ reads as

$$
\begin{aligned}
\partial_{t} n+U\left(i k_{s}+\partial_{z}\right) n+i k_{s} c_{s} w+c_{s} \partial_{z} w & =0 \\
\partial_{t} w+U\left(i k_{s}+\partial_{z}\right) w+i k_{s} c_{s} n+c_{s} \partial_{z} n & =-i \gamma_{p} k_{s} c_{s}^{-1} E_{p} \overline{E_{m}}, \\
\partial_{t} E_{m}-c_{g} \partial_{z} E_{m}+i \beta \bar{n} E_{p} & =0 .
\end{aligned}
$$

We perform a simple change of notations which allows to exhibit two simple travelling waves

$$
r=n+w, \quad s=n-w .
$$

Then, by denoting $b=-i \gamma_{p} k_{s} c_{s}^{-1} \overline{E_{m}}$, this system reads

$$
\begin{array}{r}
\partial_{t} r+U\left(i k_{s}+\partial_{z}\right) r+c_{s}\left(i k_{s}+\partial_{z}\right) r-E_{p} b=0, \\
\partial_{t} s+U\left(i k_{s}+\partial_{z}\right) s-c_{s}\left(i k_{s}+\partial_{z}\right) s+E_{p} b=0, \\
\partial_{t} b-c_{g} \partial_{z} b-\beta \gamma_{p} k_{s} c_{s}^{-1} \overline{E_{p}} \frac{r+s}{2}=0 .
\end{array}
$$

On the opposite, we consider the following system obtained by neglecting the $s$ wave:

$$
\begin{aligned}
\partial_{t} \tilde{n}+U\left(i k_{s}+\partial_{z}\right) \widetilde{n}+c_{s}\left(i k_{s}+\partial_{z}\right) \widetilde{n}-b E_{p} / 2 & =0, \\
\partial_{t} b-c_{g} \partial_{z} b-\beta \gamma_{p} k_{s} c_{s}^{-1} \overline{E_{p}} \widetilde{n} & =0,
\end{aligned}
$$

where $\widetilde{n}$ play the part of $\frac{r}{2}$ (notice that if $s$ is set to zero, $\widetilde{n}$ is reduced to $n$ ).

We want to prove that from the stability analysis, these two systems are equivalent. More precisely, we perform the Fourier transform with respect to the $z$ variable ( $\xi$ denotes the Fourier variable) for both systems and we want to prove that, for a given value of the $\xi$ variable the unstable modes of the two systems are very similar. Thus, we are looking at a solution $(r, s, b)$ of $(69)$ in the form $r(t)=\mathrm{e}^{-i \omega t} \mathcal{F}^{-1}(R(\xi)), s(t)=\mathrm{e}^{-i \omega t} \mathcal{F}^{-1}(S(\xi))$, $b(t)=\mathrm{e}^{-i \omega t} \mathcal{F}^{-1}(B(\xi))$. That corresponds to $(R, S, B)$ satisfying

$$
\begin{aligned}
\left(-\omega+\left(k_{s}+\xi\right) U\right) R+c_{s}\left(k_{s}+\xi\right) R+i E_{p} B & =0, \\
\left(-\omega+\left(k_{s}+\xi\right) U\right) S-c_{s}\left(k_{s}+\xi\right) S-i E_{p} B & =0, \\
-\omega B-c_{g} \xi B+i \beta \gamma_{p} k_{s} c_{s}^{-1} \overline{E_{p}} \frac{R+S}{2} & =0,
\end{aligned}
$$

and we look for an eigenvalue $\omega$ of this system with $\operatorname{Im}(\omega)>0$. 
In the same way, for the reduced system $(70)$, we set $\widetilde{n}(t)=\mathrm{e}^{-i \omega t} \mathcal{F}^{-1}(N(\xi)), b(t)=\mathrm{e}^{-i \omega t} \mathcal{F}^{-1}(B(\xi))$; that corresponds to the linear system

$$
\begin{aligned}
\left(-\omega+U\left(k_{s}+\xi\right)+c_{s}\left(k_{s}+\xi\right)\right) N+i B \frac{E_{p}}{2} & =0, \\
\left(-\omega-c_{g} \xi\right) B+i \beta \gamma_{p} k_{s} c_{s}^{-1} \overline{E_{p}} N & =0 .
\end{aligned}
$$

We now state the following result which is proved in Annex 1.

Proposition. Let us assume that $\xi$ is given by

$$
-c_{g} \xi=\left(U+c_{s}\right)\left(k_{s}+\xi\right) .
$$

For the system (71), there exists an eigenvalue $\omega_{\text {full }}$ such that $\operatorname{Im}\left(\omega_{\text {full }}\right)>0$; moreover, for the system (72), there exists an eigenvalue $\omega_{\text {redu }}$ such that $\operatorname{Im}\left(\omega_{\text {redu }}\right)>0$. Moreover, we have

$$
\omega_{\text {full }}=\omega_{\text {redu }}+O\left(\left|E_{p}\right|^{2}\right), \quad \text { when }\left|E_{p}\right| \text { is small. }
$$

So, with the initial system (69), the time increasing solutions are associated to a value $\omega_{\text {full }}$ which is close to the value $\omega_{r e d u}$ corresponding to the time increasing solutions of the reduced system (70). Therefore we can claim, by coming back to the notation $E_{m}$, that for a fixed value of $E_{p}$ the initial system (68) may be replaced by the reduced system

$$
\begin{aligned}
\partial_{t} E_{m}-c_{g} \partial_{z} E_{m} & =-i \beta \overline{\widetilde{n}} E_{p}, \\
\partial_{t} \widetilde{n}+\left(c_{s}+U\right)\left(i k_{s}+\partial_{z}\right) \widetilde{n} & =-\frac{i \gamma_{p} k_{s}}{2 c_{s}} E_{p} \overline{E_{m}}
\end{aligned}
$$

We now add to the above system the evolution equation for the wave $E_{p}$. We get the following standard decay system:

$$
\begin{aligned}
\partial_{t} E_{p}+c_{g} \partial_{z} E_{p} & =-i \beta \widetilde{n} E_{m}, \\
\partial_{t} E_{m}-c_{g} \partial_{z} E_{m} & =-i \beta \overline{\widetilde{n}} E_{p}, \\
\partial_{t} \widetilde{n}+\left(c_{s}+U\right)\left(i k_{s}+\partial_{z}\right) \widetilde{n} & =-\frac{i \gamma_{p} k_{s}}{2 c_{s}} E_{p} \overline{E_{m}} .
\end{aligned}
$$

Since $E_{p}, E_{m}, \widetilde{n}$ are space-time envelopes of functions which correspond to waves, it is called a three-wave coupling system.

\subsection{Analysis of the three-wave coupling system}

Notice that in this system, two very different characteristic speeds $c_{g}$ and $\left(c_{s}+U\right)$ occur, which are respectively in the order of the light speed and the sound speed. Moreover, there are quadratic coupling terms in the right hand side which correspond to the coupling between the three waves.

From now on, we restrict ourselves to the case where $\left(c_{s}+U\right)$ is positive (that is to say we do not deal with a supersonic flow in the backward direction). The simulation domain is assumed to be the half real axis or the interval $\left(z \in\left[0, z_{\max }\right]\right)$. Since we are looking at a local phenomenon in time, the incoming boundary condition for the pump wave may be assumed to be independent from the time. Then, the natural boundary conditions are the following:

$$
E_{p}(t, 0)=\alpha^{\text {in }}, \quad E_{m}\left(t, z_{\max }\right)=0, \quad \widetilde{n}(t, 0)=0 .
$$

If the initial values of $\widetilde{n}$ and $E_{m}$ are zero, then $E_{p}(t)=\alpha^{\text {in }}, E_{m}(t)=\widetilde{n}(t)=0$ is a trivial solution. For getting a non trivial solution for $(73)$, it is sufficient to set $\widetilde{n}(0,$.$) equal to a small random noise and E_{p}(0)=\alpha^{\text {in }}$, $E_{m}(0)=0$. One can also replace the previous boundary condition on $\widetilde{n}$ by $\widetilde{n}(t, 0)$ equal to a small random noise. 
The previous model may be easily extended for addressing the general case with $U$ and $N^{0}$ smooth functions which depend on $z$. Instead of (65)-(67), one considers the following system:

$$
\begin{gathered}
\partial_{t} E_{p}+c_{g} \partial_{z} E_{p}+\nu_{a} E_{p}=-i \beta M \widetilde{n} E_{m}+i \beta(1-M) E_{p} \\
\partial_{t} E_{m}-c_{g} \partial_{z} E_{m}+\nu_{a} E_{m}=-i \beta M \overline{\widetilde{n}} E_{p}+i \beta(1-M) E_{m} \\
\partial_{t} \tilde{n}+\left(c_{s}+U\right)\left(i k_{s}+\partial_{z}\right) \widetilde{n}=-\frac{i \gamma_{p} k_{s}}{2 c_{s}} E_{p} \overline{E_{m}}-c_{s} \frac{\widetilde{n}}{2} \partial_{z}\left(\log N^{0}+\frac{U}{c_{s}}\right) .
\end{gathered}
$$

Notice that in the velocity equation of system (67), a friction term may be introduced which corresponds to the so called Landau damping effect: in its simplest form it reads as $2 \nu_{L} w$ (see for instance [41]). Therefore, arguing as before, with this correction term, the last equation reads

$$
\partial_{t} \tilde{n}+\left(c_{s}+U\right)\left(i k_{s}+\partial_{z}\right) \widetilde{n}+\nu_{L} \widetilde{n}=-\frac{i \gamma_{p} k_{s}}{2 c_{s}} E_{p} \overline{E_{m}}-c_{s} \frac{\widetilde{n}}{2} \partial_{z}\left(\log N^{0}+\frac{U}{c_{s}}\right)
$$

In order to deal with this system, let us set

$$
\gamma_{0}=\left|\alpha^{\text {in }}\right| \sqrt{\frac{\beta \gamma_{p} k_{s}}{2 c_{s}}}
$$

and define the dimensionless functions $\widehat{E_{p}}, \widehat{E_{m}}, \widehat{n}$ by

$$
E_{p}=\widehat{E_{p}} \alpha^{\text {in }}, \quad E_{m}=-i \widehat{E_{m}} \alpha^{\text {in }} \sqrt{\beta}, \quad \tilde{n}=\widehat{n} \gamma_{0} \frac{1}{\sqrt{\beta}}
$$

Then, the previous system reads

$$
\begin{aligned}
\left(\partial_{t}+c_{g} \partial_{z}\right) \widehat{E_{p}}+\nu_{a} \widehat{E_{p}} & =-\gamma_{0} M \widehat{E_{m}} \widehat{n}+i \gamma_{0}(1-M) \widehat{E_{p}}, \\
\left(\partial_{t}-c_{g} \partial_{z}\right) \widehat{E_{m}}+\nu_{a} \widehat{E_{m}} & =\gamma_{0} M \widehat{E_{p}} \widehat{\widehat{n}}+i \gamma_{0}(1-M) \widehat{E_{m}} \\
\left(\partial_{t}+i k_{s}\left(c_{s}+U\right)+\left(c_{s}+U\right) \partial_{z}\right) \widehat{n}+\nu_{L} \widehat{n} & =\gamma_{0} \widehat{E_{p}} \widehat{\widehat{E_{m}}}-\frac{c_{s}}{2} \widehat{n} \partial_{z}\left(\log M+\frac{U}{c_{s}}\right) .
\end{aligned}
$$

One can check that the good characteristic length is given by $\sqrt{c_{s} c_{g}} / \gamma_{0}$, then we define the dimensionless time and space variables

$$
T=\gamma_{0} t, \quad Z=z \gamma_{0} / \sqrt{c_{s} c_{g}} .
$$

Let us introduce the small parameter $\varepsilon$ (different from the $\epsilon$ introduced previously):

$$
\varepsilon=\sqrt{c_{s} / c_{g}}
$$

and perform the change of notations:

$$
\widehat{E_{p}}(t, z)=f(T, Z), \quad \widehat{E_{m}}(t, z)=g(T, Z), \quad \widehat{n}(t, z)=\frac{1}{\varepsilon} q(T, Z) .
$$


The factor $\varepsilon^{-1}$ is introduced for normalization purpose. Then we get the following dimensionless system:

$$
\begin{aligned}
\left(\partial_{T}+\frac{1}{\varepsilon} \partial_{Z}\right) f & =-\frac{1}{\varepsilon} M g q+\frac{i}{\varepsilon}(1-M) f, \\
\left(\partial_{T}-\frac{1}{\varepsilon} \partial_{Z}\right) g & =\frac{1}{\varepsilon} M f \bar{q}+\frac{i}{\varepsilon}(1-M) g, \\
\left(\partial_{T}+i \varepsilon R+\varepsilon\left(1+\frac{U}{c_{s}}\right) \partial_{Z}\right) q+\varepsilon \nu_{l} q & =\varepsilon f \bar{g}-\frac{\varepsilon}{2} q \partial_{Z}\left(\log M+\frac{U}{c_{s}}\right),
\end{aligned}
$$

where we set

$$
\nu_{l}=\nu_{L} \gamma_{0}^{-1} \sqrt{c_{g} / c_{s}}, \quad R=k_{s} \sqrt{c_{g} c_{s}}\left(1+U c_{s}^{-1}\right) / \gamma_{0}
$$

The damping term $\nu_{l}$ corresponds to the inverse of a distance and it is small with respect to 1 ; the coefficient $R$ is the ratio of the characteristic length to the laser wave length (up to the multiplicative constant $4 \pi\left(1+U c_{s}^{-1}\right)$ ). This system is supplemented with the boundary conditions

$$
f(T, 0)=f^{\text {in }}=1, \quad g\left(T, Z_{\max }\right)=g^{b}, \quad q(T, 0)=0
$$

where $g^{b}$ may be zero or a function of $T$ which is small compared to 1 ; of course initial conditions have also to be prescribed, for instance

$$
f(0, .)=f^{\text {in }}=1, \quad g(0, .)=0,
$$

and $q(0,$.$) a function whose L^{\infty}$ norm is small compared to 1 . Notice that if $g^{b}=0$ and $q(0,)=$.0 , we get a trivial stationary solution: $f=\exp (i(1-M) Z), g=0$ and $q=0$.

Remark 1. Conservation properties.

If $g^{b}=0$, it is easy to check that the solution of system (78)-(80) satisfies:

$$
\partial_{T}\left(\int_{0}^{Z_{\max }}|f|^{2} \mathrm{~d} Z+\int_{0}^{Z_{\max }}|g|^{2} \mathrm{~d} Z\right)+\left|f_{Z_{\max }}\right|^{2}+\left|g_{0}\right|^{2}=\left|f^{\mathrm{in}}\right|^{2},
$$

On the other hand, one can check that

$$
\partial_{T}\left(\varepsilon^{2}|f|^{2}\right)+M \partial_{T}\left(|q|^{2}\right)+\varepsilon \partial_{Z}|f|^{2}+\varepsilon M \partial_{Z}\left(|q|^{2}\left(1+\frac{U}{c_{s}}\right)\right)+2 \nu_{l}|q|^{2}=-\varepsilon|q|^{2} \partial_{Z} M,
$$

then, if $\nu_{l}=0$ and if $M$ is constant, there is another conservation relation:

$$
\partial_{T}\left(\varepsilon^{2} \int_{0}^{Z_{\max }}|f|^{2} \mathrm{~d} Z+\int_{0}^{Z_{\max }} M|q|^{2} \mathrm{~d} Z\right)+\varepsilon^{2}\left|f_{Z_{\max }}\right|^{2}+M\left(1+\frac{U_{Z_{\max }}}{c_{s}}\right)\left|q_{Z_{\max }}\right|^{2}=\varepsilon^{2}\left|f^{\text {in }}\right|^{2} .
$$

Remark 2. If we set $M=1, \nu_{l}=0$ and $U$ constant, this system may read in a more esthetic formulation after a new change of variables:

$$
\begin{aligned}
\left(\partial_{T}+\frac{1}{\varepsilon} \partial_{Z}\right) a_{0} & =-\frac{1}{\varepsilon} \overline{a_{1} a_{2}}, \\
\left(\partial_{T}-\frac{1}{\varepsilon} \partial_{Z}\right) a_{1} & =\frac{1}{\varepsilon} \overline{a_{0} a_{2}}, \\
\left(\partial_{T}+\varepsilon \partial_{Z}\right) a_{2} & =\varepsilon \overline{a_{1} a_{0}} .
\end{aligned}
$$

This system has been studied for a long time by the physicists (its derivation and properties are explained in [38]). But up to our knowledge, the mathematical analysis of this system (either on a half real axis or on a finite 
interval) is still an open problem. Annex 1 is devoted to some preliminary remarks and conjectures related to this problem.

\subsection{On the numerical techniques}

From the numerical point of view, there are two main difficulties for solving the system (78)-(80): first, it involves two different characteristic speeds (the sound speed and the light speed); second, two waves travel one way and the third one travel in the opposite direction. It looks like that in physicist's papers, the numerical schemes for dealing with the advection terms in the three equations are explicit in time. So it leads to time steps in the order of $\delta x / c_{g}$ (which are very small compared to the time scale of the phenomena) and to a prohibitive CPU time. The aim of this subsection is to give some features on the numerical techniques of the implicit type (at least for the two laser wave equations) and to enlighten the reader on the related numerical difficulties.

Let us define

$$
\chi(Z)=1+U(z) / c_{s}, \quad H=-\frac{1}{2} \partial_{z}(\log M)-\frac{1}{2} \partial_{z}\left(\frac{U}{c_{s}}\right)+\nu_{l} .
$$

The function $\chi$ is in the order of 1 and may change sign. As it was pointed out, the damping term $\nu_{l}$ is small with respect to 1 . According to the normalization of the spatial variable, the term $\partial_{z}(\log M)+\partial_{z}\left(\frac{U}{c_{s}}\right)$ is always small with respect to $R$, so the modulus of $H$ is negligible with respect to $R$.

Then, the system to be solved reads

$$
\begin{array}{r}
\left(\partial_{T}+\frac{1}{\varepsilon} \partial_{Z}\right) f=-\frac{1}{\varepsilon} M g q+\frac{i}{\varepsilon}(1-M) f, \\
\left(\partial_{T}-\frac{1}{\varepsilon} \partial_{Z}\right) g=\frac{1}{\varepsilon} M f \bar{q}+\frac{i}{\varepsilon}(1-M) g, \\
\left(\partial_{T}+\varepsilon \chi \partial_{Z}\right) q+\varepsilon(H+i R) q=\varepsilon f \bar{g},
\end{array}
$$

with the initial conditions

$$
f(0, .)=1, \quad g(0, .)=0, \quad q(0, .) \text { given and small (with respect to } 1),
$$

and the boundary conditions

$$
\begin{aligned}
f(T, 0) & =1, \quad \forall T, \\
g\left(T, Z_{\max }\right) & =g^{b}(T), \forall T, \\
q(T, 0) & =0, \quad \forall T, \quad \text { if } \chi(0)>0 .
\end{aligned}
$$

Notice that if $\chi(0)<0$, there is no boundary condition for $q(T, 0)$. For the sake of simplicity, we assume that $\chi\left(Z_{\max }\right)>0, \chi(0)>0$.

\section{Numerical characteristic sizes}

Usually, for hot plasmas (whose temperature are in the order of some $10^{7}$ Kelvin), the parameter $\varepsilon$ ranges in the interval $[0.03,0.04]$. Moreover, the characteristic length $\sqrt{c_{s} c_{g}} / \gamma_{0}=c_{s} /\left(\varepsilon \gamma_{0}\right)$ depends on the incoming laser intensity, the non dimensional density $N_{\text {ref }}$ and physical characteristic of the plasma; typically, it ranges from 8 wave lengths $\left(2 \pi k_{0}^{-1}\right)$ to 30 wave lengths (or more if the incoming laser intensity is small). One checks that typically, $R \in[100,400]$.

In order to study the numerical properties of the scheme we will consider the previous problem in the case where the density $M$ and the velocity $U$ are constant (even if it is not very realistic from a physical point of view), then $H=\nu_{l}$. We address simulations on a spatial domain with $Z_{\max }$ much larger than some units and for $T$ in the order of 100 at least. 
For an efficient numerical treatment, we wish to use for this model a spatial step which is not too small compared to the one used in the paraxial code (indeed one solves the hydrodynamic system coupled to this model through the ponderomotive force), that is to say $\delta Z$ ranges in the intervall $[0.03,0.3]$.

As usual, we expect to get a numerical method which is stable with time steps $\delta T$ as large as possible. Then the time discretization of equations (81), (82) has to be implicit with respect to the time variable in the same way as in Section 3.4 for avoiding time steps smaller than $\varepsilon \delta Z$.

Denote by $f_{j}^{n}, g_{j}^{n}, q_{j}^{n}$ the values of $f, g, q$ evaluated at time $T^{n}=n \delta T$ and position $Z_{j}=j \delta Z$. We now describe a numerical method in the particular case, where $M=1$ (it is easy to generalize to the case where $M$ is variable).

We assume that all the values are known at time $T^{n-1}$ and we look for the values at time $T^{n}$. We address first the laser field equations (81), (82). To obtain the quantities $f_{j}^{n}$, we use a classical space marching technique from the boundary condition corresponding to $j=0$. To deal with the source term in the right hand side of equation (81), we write $g_{j}^{n-1} q_{j}^{e x}$ where $q_{j}^{e x}$ is an extrapolated value of $q_{j}$ over a time step obtained according to $(83)$, that is to say

$$
q_{j}^{e x}=q_{j}^{n-1}+\varepsilon \delta T f_{j}^{n} \overline{g_{j}^{n-1}} .
$$

Thus, the space marching equation reads

$$
\frac{\varepsilon}{\delta T}\left(f_{j-1}^{n}-f_{j-1}^{n-1}\right)+\frac{1}{\delta Z}\left(f_{j}^{n}-f_{j-1}^{n}\right)=-g_{j}^{n-1} q_{j}^{n-1}-\varepsilon \delta T\left|g_{j}^{n-1}\right|^{2} f_{j}^{n},
$$

which solution is straightforward by travelling from the incoming boundary $(j=1)$. By the same way, we use a space marching technique in the backward direction to get the value $g_{j}^{n}$. Using the updated value $f_{*}^{n}$, we deal here with the source term by writting $q_{j}^{e x}=q_{j}^{n-1}+\varepsilon \delta T f_{j}^{n} \overline{g_{j}^{n}}$; so, by travelling from the rear boundary $\left(j=j_{\max }\right)$, we have to solve:

$$
\frac{\varepsilon}{\delta T}\left(g_{j+1}^{n}-g_{j+1}^{n-1}\right)+\frac{1}{\delta Z}\left(g_{j}^{n}-g_{j+1}^{n}\right)=f_{j}^{n} \overline{q_{j}^{n-1}}+\varepsilon \delta T\left|f_{j}^{n}\right|^{2} g_{j}^{n} .
$$

Afterwards, the third equation (83) is solved in a classical explicit way with the source term evaluated with the help of the updated values $f^{n}$ and $g^{n}$. It reads as

$$
\begin{array}{cr}
\left(q_{j}^{n}-q_{j}^{n-1}\right)+\varepsilon \frac{\delta T}{2}\left(i R+H_{j}\right)\left(q_{j}^{n}+q_{j}^{n-1}\right)=-\varepsilon \frac{\delta T}{\delta Z} \chi_{j}\left(q_{j+1 / 2}^{n-1}-q_{j-1 / 2}^{n-1}\right)+\delta T \varepsilon f_{j}^{n} \overline{g_{j}^{n}}, & \begin{array}{r}
q_{j+1 / 2}^{n-1}=q_{j}^{n-1} \\
\text { if } \chi_{j}>0 ;
\end{array} \\
q_{j+1 / 2}^{n-1}=q_{j+1}^{n-1}, & \text { else. }
\end{array}
$$

Notice that for a accurate time discretization of the phase term $(i \varepsilon R)$ for $q$, one must have

$$
\delta T \preceq(\varepsilon R)^{-1} .
$$

We will see in numerical examples that this condition on $\delta T$ is not very restrictive.

Remark (modified method). As a matter of fact, in order to have a numerical method wich is compatible with the method described in Section 4.4.1 and which has good energy balance properties, we split each step of the space marching algorithmus in two sub-steps.

Let us focus on the main laser wave $f$. To switch from $f_{j-1}^{n}$ to $f_{j}^{n}$, the first sub-step consists in solving

$$
\frac{\varepsilon}{\delta T}\left(f_{j-1}^{n}-f_{j-1}^{n-1}\right)+\frac{1}{\delta Z}\left(\widetilde{f_{j}^{n}}-f_{j-1}^{n}\right)=0,
$$


which is done by computing separately the square of the modulus and the argument as in Section 4.4.1. The second sub-step consists in stating

$$
\frac{1}{\delta Z}\left(f_{j}^{n}-\widetilde{f_{j}^{n}}\right)=-g_{j}^{n-1} q_{j}^{n}-\varepsilon \delta T\left|g_{j}^{n-1}\right|^{2} f_{j}^{n}
$$

Concerning the backscattered wave $g$, to switch from $g_{j+1}^{n}$ to $g_{j}^{n}$, the first sub-step consists in solving

$$
\frac{\varepsilon}{\delta T}\left(g_{j+1}^{n}-g_{j+1}^{n-1}\right)+\frac{1}{\delta Z}\left(\widetilde{g_{j}^{n}}-g_{j+1}^{n}\right)=0
$$

which is solved by computing separately the square of the modulus and the argument. The second sub-step consists in stating

$$
\frac{1}{\delta Z}\left(g_{j}^{n}-\widetilde{g_{j}^{n}}\right)=f_{j}^{n} \overline{q_{j}^{n-1}}+\varepsilon \delta T\left|f_{j}^{n}\right|^{2} g_{j}^{n}
$$

\subsection{Numerical results}

We have made some computations for checking the stability of the numerical schemes, for different values of $\delta Z$ and $\delta T$.

In all the numerical tests, we have set $\varepsilon=0.04$. The characteristic length $c_{s} /\left(\varepsilon \gamma_{0}\right)$ is equal to 8 wave lengths so we have $R=100$. The size of the domain is equal to $Z_{\max }=10$ (which corresponds to 28 microns for a standard wave length of 0.35 microns).

As it has been noticed the initial and boundary values for $q$ and $g$ are somehow random. Two series of computations have been performed. The first ones with a zero initial value for $q$, but with a small boundary condition $g^{b}$ on the right hand side of computational domain; in that case this data is given by the following function which is supposed to play the part of a small random function and to excite numerous time modes:

$$
g^{b}(T)=\beta\left[0.3 \mathrm{e}^{12 i T}+0.3 \mathrm{e}^{i(2 T+1.5)}-0.5 \mathrm{e}^{i(4 T+1)}+0.2 \mathrm{e}^{i(7 T+2)}\right], \quad \beta \text { constant }
$$

The second ones with the following initial value for $q$ which is also supposed to play the part of a small random function

$$
q(0, Z)=c_{i n i} \frac{Z^{2}}{(0.3 L+Z)^{2}}[0.6 \exp (4 i Z)+0.4 \exp (i(Z+2.5))+0.2 \exp (15 i Z)],
$$

with $c_{i n i}=0.03$ and with the initial and boundary values of $g$ which are zero.

All the computations have been performed with a small value of $\nu_{l}$, which is $\nu_{l}=0.05$ (if this value is larger, the stability of the numerical method will be easier to obtain).

In the sequel the reflectivity $\mathcal{R}(T)$ is defined by

$$
\mathcal{R}(T)=|g(T, 0)|
$$

It corresponds to the square root of the energy which will be reflected by the plasma due to the Brillouin instability. Since the plasma is homogeneous without longitudinal velocity and since the damping coefficient $\nu_{l}$ is very small, the reflectivity may increase up to 1 when the time $T$ is large enough.

\section{Boundary value for $g$.}

We first choose $\beta=10^{-4}$. The time step is fixed to $\delta T=0.09$ and we compare the solutions for $\delta Z=0.3$ and $\delta Z=0.1$; we show in Figure 4, the profiles of the function $|f|$ and $|g|$ at ten regularly spaced times from $T=15$ up to $T=150$ and at the final time $T=200$. In Figure 5 , we plot the reflectivity versus the time for both cases. 

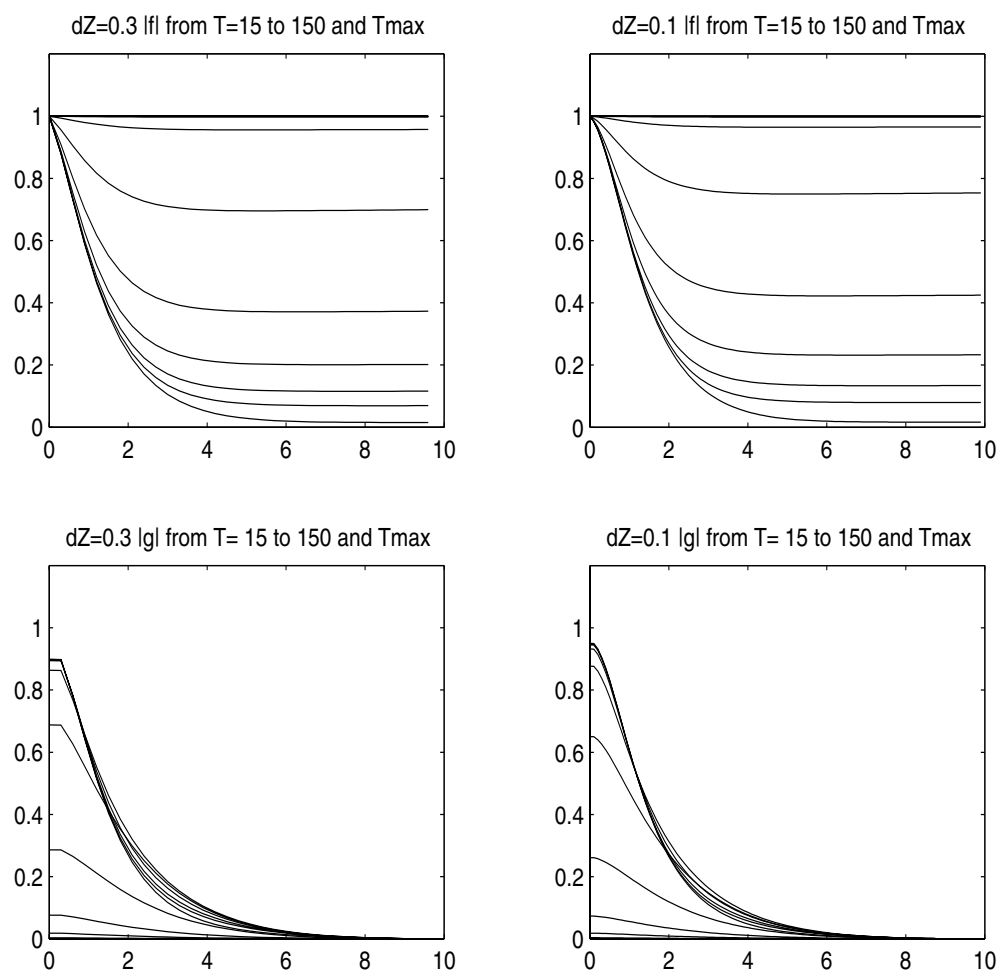

Figure 4. (Above) Profiles of $|f(T,)$.$| versus Z$, at different times, for two values of the space step $\delta Z=0.3$ (left side) and $\delta Z=0.1$ (right side)[when time increases, the profiles decrease]. (Bottom) Same curves for $|g(T,)$.$| [when time increases, the profiles increase]. Simulation with$ a non zero boundary condition on $g$.

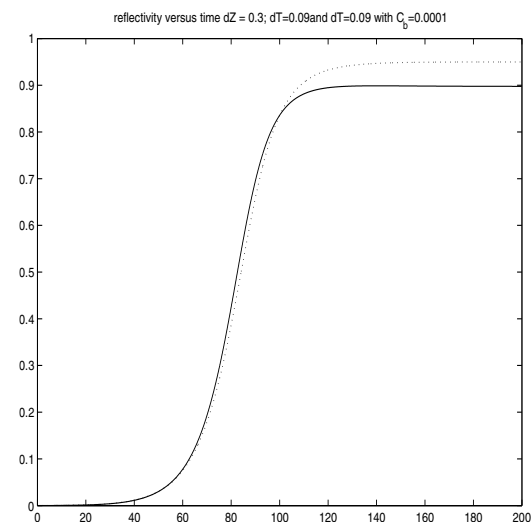

FiguRE 5. Reflectivity of laser intensity versus time, for two values of the space step $\delta Z=0.3$ (plain line) and $\delta Z=0.1$ (dotted line). Simulation with a non zero boundary condition on $g$.

Notice that the results are identical if we take $\delta T=0.12$ but by choosing a larger value of $\nu_{l}$, for instance $\nu_{l}=0.2$. (If we take a time step $\delta T=0.12$, and $\nu_{l}=0.05$, the numerical scheme may become unstable when $T$ is large enough, say in the order of 150 or larger). 


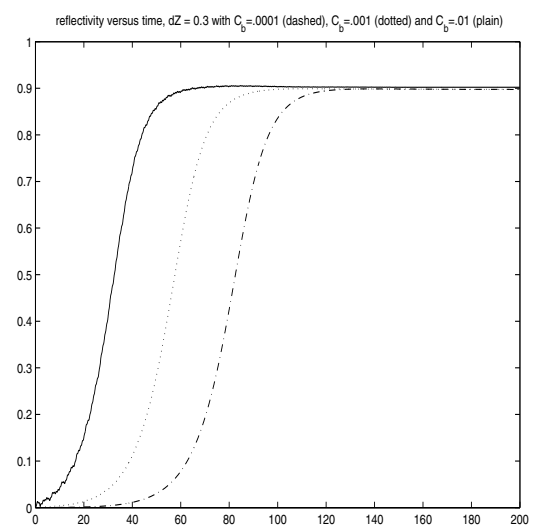

FIGURE 6. Reflectivity of laser intensity versus time for 3 value of the boundary coefficient $c_{b}=0.0001$ (dot-dashed line), $=0.001$ (dotted line),$=0.01$ (plain line).

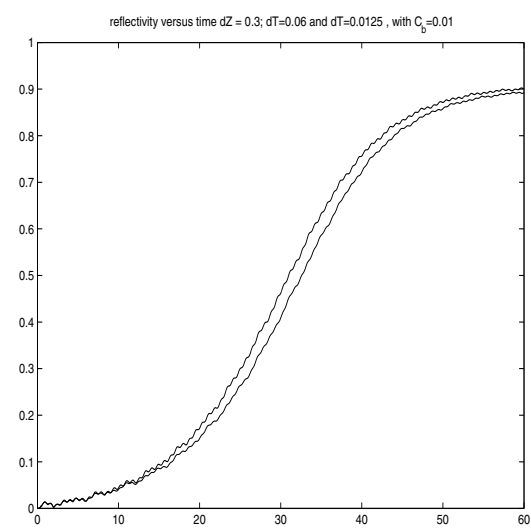

Figure 7. Comparison of the reflectivity with $\delta T=0.06$ (zoom of the previous figure) and $\delta T=0.0125$.

Of course, the results with $\delta Z=0.1$ are more accurate than those with larger $\delta Z$, but the results show the same phenomenology: the reflectivity grows exponentionaly fast at the begining of the simulation, and afterwards, a saturation occurs and the reflectivty is stabilized at a level near 1 . The maximum level of reflectivty depends of course on the space step, but the results are quite accurate even with $\delta Z=0.3$. Let us now modify the value of the coefficient $\beta$. In Figure 6 , we plot the reflectivity versus time for three computations corresponding to $\beta=10^{-4}, \beta=10^{-3}, \beta=10^{-2}$, all with $\delta Z=0.3, \nu_{l}=0.05, \delta T=0.09$ (except for the case $\beta=10^{-2}$ where $\delta T$ has been fixed to 0.06 in order to avoid numerical instabilities for large values of the time $T)$.

We check that the three curves has the same general shape, but they are shifted with respect to time.

The oscillations in the curves for the reflectivity corresponding to $\beta=10^{-2}$ are not a numerical artefact, they are due to the amplification of the oscillation of the boundary condition $g^{b}$. Indeed we have performed another computation with a very small time step $\delta T=0.0125$ (which corresponds roughly to the Courant condition for the light velocity $1 / \varepsilon$ ) and the results are quite identical as it may be seen in Figure 7 . The two curves are very close up to $T=12$ and after that time, only a little time shift between the two curves may be observed. 

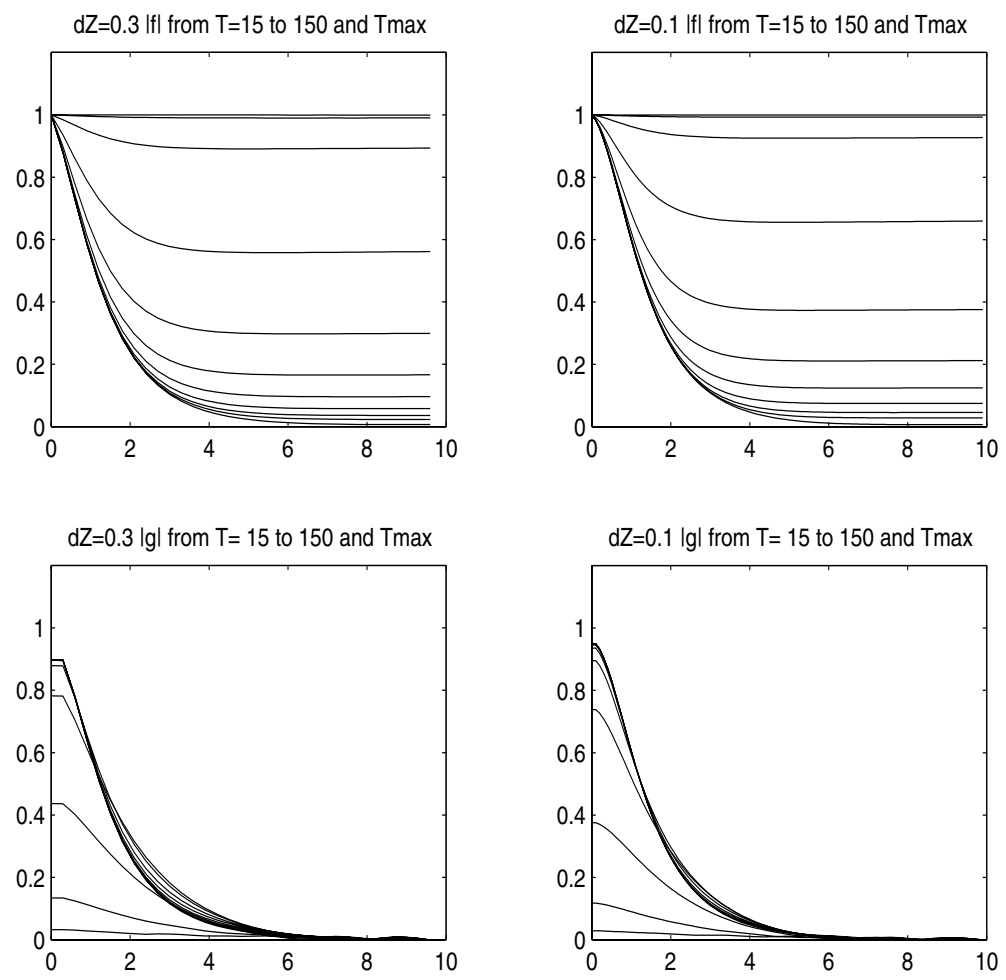

Figure 8. (Above) Profiles of $|f(T,)$.$| versus Z$, at different times, for two values of the space step $\delta Z=0.3$ and $\delta Z=0.1$ [when time increases, the profiles decrease]. (Bottom) Same curves for $|g(T,)$.$| [when time increases, the profiles increase]. Simulation with a non zero initial$ condition on $q$.

\section{Initial value for $q$.}

Let us address the problem with the initial value mentionned above. We choose $\delta T=0.09$ and we compare the solutions for $\delta Z=0.3$ and $\delta Z=0.1$.

In Figure 8, the profiles of the function $|f|$ and $|g|$ are plotted at regularly spaced times. In Figure 9, we plot the reflectivity versus time for both cases.

Notice that the shape of the reflectivity curves is the same as in the case of the boundary value problem (with $q(0,$.$) set to zero). When the time step decreases, the profile of the different curves remains very similar.$

But as previously, if one increases the time step the numerical scheme becomes unstable at a level which is quite the same with the two space steps. The criterium of stability depends mainly on the value of the damping coefficient $\nu_{l}$.

Comments. These numerical simulations with the chosen value of $\nu_{l}$, lead to think that the proposed numerical method is stable if the dimensionless time step $\delta T$ is less than a maximal value which is in the order of $1 / 10$ (notice that if $\nu_{l}$ were zero, this constant would be smaller and if $\nu_{l}$ increased, this constant would be larger).

Let us go back to the physical quantities, recall that $\sqrt{c_{s} c_{g}} \delta t / \delta z=\delta T / \delta Z$. Recall that the ratio between the characteristic length of the three-wave coupling $\left(\sqrt{c_{s} c_{g}} \gamma_{0}^{-1}\right)$ over the laser wave length may range in the interval $[8,30]$.

If one wishes to have a good and efficient simulation which takes into account the standard decay system, the diffraction phenomena as in equations (65), (66) and a basic hydrodynamics system (61), (62), the spatial step must be as large as possible but smaller than 2 or 3 wave lengths (for the diffraction treatment) and smaller 


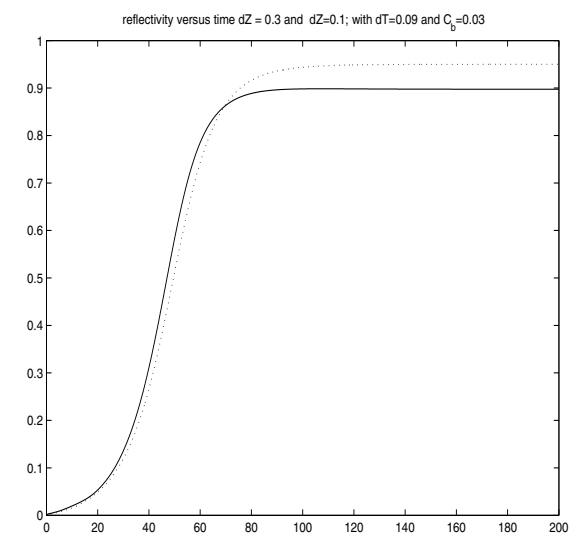

FIgURE 9. Reflectivity of laser intensity versus time, for two values of the space step $\delta Z=0.3$ (plain line) and $\delta Z=0.1$ (dashed line). Simulation with a non zero initial condition on $q$.

than a fraction of the characteristic length, that is to say $\delta Z$ has to be in the order of 0.25 or less (when the coupling is weak). Thus there exists a constant $C_{0}$ such that the proposed numerical treatment of the three-wave coupling system prescribes that

$$
\sqrt{c_{s} c_{g}} \delta t / \delta z \leq C_{0}, \quad \text { where } C_{0} \in[0.4,1]
$$

Since $\sqrt{c_{s} / c_{g}} \simeq 0.04$, this leads to a crtierium

$$
c_{s} \delta t / \delta z \leq 0.04 C_{0}
$$

Compared to the standard hydrodynamics CFL criterium which may read as $c_{s} \delta t / \max (\delta z, \delta x, \delta y) \leq C_{H}$ with $C_{H}$ in the order of 1 , criterium (85) is somehow restrictive. But notice that this criterium would have been replaced by

$$
c_{s} \delta t / \delta z \leq(0.04)^{2}
$$

if we had used explicit schemes for solving the three equations of the system (since the stability criterium would have been related to the light speed).

\section{Conclusion}

Many length scales and time scales are to be taken into account in the physics of the laser-plasma interaction. If one wished to perform simulations on a sufficient large domain during a time long enough by solving the full Maxwell equations and the two-component Euler system for ions and electrons of the plasma, it would lead to prohibitive CPU time. So physicists generally use approximated models. In this paper, we focused on the coupling of the laser propagation and the ion acoustic waves. So, from the Euler-Maxwell system, we have first derived the so called basic time envelope model obtained by a classical average of all the quantities over a laser period.

In Section 3, by using the W.K.B. approximation, we have recalled the main properties of the geometrical optics model and the difficulties related to the possible existence of a caustic surface.

In the following section we have emphasized the different assumptions which are necessary to derive the paraxial approximation. This last model is intensively used by physicists but its validity has to be checked carefully. We have stated the paraxial equation in a general tilded frame when the incidence angle between the laser direction and the gradient of the plasma density is not zero. The classical paraxial equation is a particular case corresponding to a zero incidence angle. With massively parallel computer architectures, it is now possible 
to perform 3D computations based on the paraxial approximation on a reasonable space and time domain if one deals with implicit time scheme for the laser propagation equation.

In the last section, we have stated the standard decay model for the simulation of the Brillouin instability; it consists in a three-wave coupling system: the first two waves are the pump laser wave and the backscattered laser wave which travel at the light speed and the third one is the acoustic wave which travels at the sound speed. This system has been studied for a long time, but its numerical treatment is very challenging if one wants to use time steps much larger than the one given by the Courant criterium related to light speed. On the other hand, a mathematical analysis of this system is lacking and the statement of some mathematical properties would surely help to devise an efficient numerical scheme for this system.

\section{Annex 1. Proof of the proposition of Section 5.1 for the three-Wave Coupling SYSTEM}

The characteristic polynomial of the system (71) reads as

$$
\left(\omega+c_{g} \xi\right)\left(\left(\omega-\left(k_{s}+\xi\right) U\right)^{2}-c_{s}^{2}\left(k_{s}+\xi\right)^{2}\right)+\beta \gamma_{p} k_{s}\left|E_{p}\right|^{2}\left(k_{s}+\xi\right)=0
$$

Notice that if $E_{p}=0$, this equation has three real roots

$$
\omega_{1}=\left(k_{s}+\xi\right)\left(U+c_{s}\right), \quad \omega_{2}=\left(k_{s}+\xi\right)\left(U-c_{s}\right), \quad \omega_{3}=-c_{g} \xi .
$$

Thus, in order to have a non real root $\omega$ when $E_{p}$ is non zero and small, it is necessary that two of these roots mingle together in the case $E_{p}=0$. We can check that these two roots must be $\omega_{1}$ and $\omega_{3}$, so it is necessary that

$$
\xi=-k_{s} \frac{c_{s}+U}{c_{s}+U+c_{g}},
$$

which is the value stated in the proposition. By setting $\kappa=k_{s} c_{g}\left(c_{g}+U+c_{s}\right)^{-1}$, the characteristic polynomial may read as:

$$
\left(\omega-\kappa\left(U+c_{s}\right)\right)^{2}\left(\omega+\kappa c_{s}-\kappa U\right)+\beta \gamma k_{s}\left|E_{p}\right|^{2} \Gamma=0 .
$$

We now search a root $\omega$ which is close to $\omega_{3}=\kappa\left(U+c_{s}\right)$. Let us make the change of variable:

$$
\omega=\kappa\left(U+c_{s}\right)+\kappa c_{s}\left(\zeta+i \zeta_{1}\right), \text { with } \zeta, \zeta_{1} \text { real. }
$$

Then, the problem is to find a root $\left(\zeta+i \zeta_{1}\right)$ of the following equation whose absolute value is small (when $\left|E_{p}\right|$ is small):

that is to say

$$
\left(\zeta+i \zeta_{1}\right)^{2}+\zeta+2+i \zeta_{1}=-\beta \gamma k_{s}\left|E_{p}\right|^{2} \kappa^{-2} c_{s}^{-3},
$$

$$
\begin{aligned}
(2+\zeta)\left(\zeta^{2}-\zeta_{1}^{2}\right)-2 \zeta_{1}^{2} \zeta & =-\beta \gamma k_{s}\left|E_{p}\right|^{2} \kappa^{-2} c_{s}^{-3} \\
4 \zeta+3 \zeta^{2} & =\zeta_{1}^{2}
\end{aligned}
$$

Then, after some calculus, we get

$$
\zeta_{1}= \pm \sqrt{\frac{\beta \gamma_{p}}{2 k_{s} c_{s}^{3}}}\left|E_{p}\right|+O\left(\left|E_{p}\right|^{3}\right) \text { and } \zeta=\frac{\zeta_{1}^{2}}{4}+O\left(\left|E_{p}\right|^{4}\right) .
$$

Therefore, there exists a root $\omega=\omega_{\text {full }}$ of (86) such that $\operatorname{Im}\left(\omega_{\text {full }}\right)>0$, for small values of $\left|E_{p}\right|$, it reads

$$
\omega_{f u l l}=\left(k_{s}\left(U+c_{s}\right)+i \sqrt{\frac{\beta \gamma_{p} k_{s}}{2 c_{s}}}\left|E_{p}\right|+\frac{\beta \gamma_{p}}{8 c_{s}^{2}}\left|E_{p}\right|^{2}\right) \frac{c_{g}}{c_{g}+U+c_{s}}+O\left(\left|E_{p}\right|^{3}\right),
$$


and satisfies $\operatorname{Im}(\omega)>0$.

Consider now the linear system (72)

$$
\begin{aligned}
\left(-\omega+U\left(k_{s}+\xi\right)+c_{s}\left(k_{s}+\xi\right)\right) N+i B \frac{E_{p}}{2} & =0, \\
\left(-\omega-c_{g} \xi\right) B+i \beta \gamma_{p} k_{s} c_{s}^{-1} \overline{E_{p}} N & =0,
\end{aligned}
$$

and the corresponding eigenvalues $\omega_{r e d u}$ for $\xi=-k_{s} \frac{U+c_{s}}{U+c_{s}+c_{g}}$ are solutions to the characteristic polynomial

$$
\left(\omega-\kappa\left(c_{s}+U\right)\right)^{2}+\beta \gamma_{p} k_{s}\left|E_{p}\right|^{2} /\left(2 c_{s}\right)=0,
$$

thus we get

$$
\omega_{\text {redu }}=\left(k_{s}\left(c_{s}+U\right)+i \sqrt{\frac{\beta \gamma_{p} k_{s}}{2 c_{s}}}\left|E_{p}\right|\right) \frac{c_{g}}{c_{g}+U+c_{s}}+O\left(\left|E_{p}\right|^{2}\right),
$$

Then, the eigenvalues $\omega_{f u l l}$ and $\omega_{\text {redu }}$ are equal up to a second order term (in $\left|E_{p}\right|$ ).

\section{Annex 2. Mathematical PRoperties on the three-WAVE COUPling System}

We focus here on some elementary mathematical properties on a simplified system without damping term in order to understand the structure of the coupling phenomena. We assume that the constant velocity $U$ is zero (if it is not the case the calculus are the same after a slight modification of the definition of $\varepsilon$ ). By setting

$$
f=\exp (-i \varepsilon R(T-Z \varepsilon)) \overline{a_{0}}, \quad g=\exp (-i \varepsilon R(T+Z \varepsilon)) a_{1}, \quad h=\exp (i 2 \varepsilon R T) a_{2},
$$

we can transform the initial system (78),(79),(80) into the following one:

$$
\begin{aligned}
\left(\partial_{T}+\frac{1}{\varepsilon} \partial_{Z}\right) a_{0} & =-\frac{1}{\varepsilon} \overline{a_{1} a_{2}}, \\
\left(\partial_{T}-\frac{1}{\varepsilon} \partial_{Z}\right) a_{1} & =\frac{1}{\varepsilon} \overline{a_{0} a_{2}}, \\
\left(\partial_{T}+\varepsilon \partial_{Z}\right) a_{2} & =\varepsilon \overline{a_{1} a_{0}} .
\end{aligned}
$$

We address this problem with the following simplified boundary and initial conditions:

$$
\begin{aligned}
a_{0}(T, 0) & =1, \quad a_{1}\left(T, Z_{\max }\right)=0, \quad a_{2}(T, 0)=0, \quad \forall T, \\
a_{0}(0, .) & =a_{0}^{\text {ini }}, \quad a_{1}(0, .)=0 .
\end{aligned}
$$

$a_{0}^{i n i}$ is a given continuous function in $L^{2}\left(\left[0, Z_{\max }\right]\right.$ ) (whose value in $Z=0$ is equal to 1 ) and $a_{2}(0,$.$) a function$ whose $L^{\infty}$ norm is small compared to 1 . Notice first that if $a_{2}(0,)=$.0 , we get a trivial solution with $a_{1}(T,)=.a_{2}(T,)=$.0 .

By multiplying (87) by $\overline{a_{0}}$ and (88) by $\overline{a_{1}}$ and combining with the conjugate quantities, we check that

$$
\partial_{T}\left(\left|a_{0}\right|^{2}+\left|a_{1}\right|^{2}\right)+\frac{1}{\varepsilon} \partial_{Z}\left(\left|a_{0}\right|^{2}-\left|a_{1}\right|^{2}\right)=0 .
$$

Moreover, by multiplying (89) by $\overline{a_{2}}$ and (88) by $\overline{a_{1}}$, we get

$$
\partial_{T}\left(\left|a_{2}\right|^{2}+\varepsilon^{2}\left|a_{0}\right|^{2}\right)+\partial_{Z}\left(\varepsilon\left|a_{0}\right|^{2}+\varepsilon\left|a_{2}\right|^{2}\right)=0 .
$$

We now address the previous problem on the domain $\mathbf{R}^{+}$that is to say $Z_{\max }=+\infty$ and the boundary condition is $\lim _{Z=\infty} a_{1}(Z)=0$. 
Lemma. There exists a solution $\xi \in L^{2}(0,+\infty, \mathbf{R})$ of the ordinary differential equation

$$
\frac{\partial^{2}}{\partial Z^{2}}(\log \xi)=-|\xi|^{2}, \quad \xi(0)=1,\left.\quad \partial_{Z}(\log \xi)\right|_{0}=0
$$

and $a_{0}^{s}=\xi, \quad a_{1}^{s}=\xi, \quad a_{2}^{s}=-\partial_{Z}(\log \xi)$ is a stationary solution to the system (87)-(89). We have

$$
0 \leq a_{k}^{s} \leq 1, \quad \lim _{Z=\infty} a_{0}^{s}(Z)=\lim _{Z=\infty} a_{1}^{s}(Z)=0, \quad \lim _{Z=\infty} a_{2}^{s}(Z)=1 .
$$

Proof. By setting $w=-\log \xi$, the system may be written as $w^{\prime \prime}-\mathrm{e}^{-2 w}=0$ with $w(0)=w^{\prime}(0)=0$. Thus, we get $\left(\left|w^{\prime}\right|^{2}\right)^{\prime}=-\left(\mathrm{e}^{-2 w}\right)^{\prime}$. According to the boundary conditions, that implies $d w=\sqrt{1-\mathrm{e}^{-2 w}} \mathrm{~d} Z$ and there exists an increasing function $G$ such that

$$
Z=\int_{0}^{w} \frac{\mathrm{d} \lambda}{\sqrt{1-\mathrm{e}^{-2 \lambda}}}=\int_{1}^{\exp w} \frac{\mathrm{d} \mu}{\sqrt{\mu^{2}-1}}=G(w) .
$$

One can easily check that when $Z \rightarrow+\infty, w(Z)=G^{(-1)}(Z)$ tends to $+\infty$ and $w^{\prime}(Z)$ tends to 1 .

We now conjecture that if the initial data $a_{2}^{\text {ini }}$ is small enough, there exists a solution $\left(a_{0}, a_{1}, a_{2}\right)$ such that

* the modulus of $a_{0}, a_{1}, a_{2}$ are bounded uniformly in time;

* the solution $\left(a_{0}, a_{1}, a_{2}\right)$ converges to $\left(a_{0}^{s}, a_{1}^{s}, a_{2}^{s}\right)$ when $T$ goes to infinity;

* $\left|a_{1}(T, 0)\right|<1$, for any $T>0$;

* the functions $a_{0}(),. a_{1}(),. a_{2}($.$) are continuous from \mathbf{R}^{+}$into $L_{l o c, Z}^{1}$.

Acknowledgements. I wish to thank M. Casanova, S. Hüller, D. Pesme, P. Loiseau, O. Morice and F. Golse for fruitful discussions on these problems.

\section{REFERENCES}

[1] M.R. Amin, C.E. Capjack, P. Fricz, W. Rozmus and V.T. Tikhonchuk, Two-dimensional studies of stimulated Brillouin scattering, filamentation. Phys. Fluids B 5 (1993) 3748-3764.

[2] A. Arnold and M. Ehrhardt, Discrete transparent boundary conditions for wide angle parabolic equations. J. Comput. Phys. 145 (1998) 611-638.

[3] P. Ballereau, M. Casanova, F. Duboc, D. Dureau, H. Jourdren, P. Loiseau, J. Metral, O. Morice and R. Sentis, Coupling hydrodynamics with a paraxial solver for laser propagation. CEA internal report (2005).

[4] J.D. Benamou, An introduction to Eulerian geometrical optics. J. Sci. Comp. 19 (2003) 63-95.

[5] J.D. Benamou, F. Castella, T. Katsaounis and B. Perthame, High Frequency limit of the Helmholtz equations. Rev. Mat. Iberoamericana 18 (2002) 187-209.

[6] J.D. Benamou, O. Lafitte, R. Sentis and I. Solliec, A geometrical optics based numerical method for high frequency electromagnetic fields computations near fold caustics (part I). J. Comput. Appl. Math. 156 (2003) 93-125.

[7] J.D. Benamou, O. Lafitte, R. Sentis and I. Solliec, A geometrical optics based numerical method for high frequency electromagnetic fields computations near fold caustics (part II, the Energy). J. Comput. Appl. Math. 167 (2004) 91-134.

[8] J.P. Berenger. A perfectly matched layer for the absorption of electromagnetic waves. J. Comput. Phys. 114 (1994) 185-200.

[9] R.L. Berger, C.H. Still, E.A. Williams and A.B. Langdon, On the dominant subdominant behavior of stimulated Raman and Brillouin scattering. Phys. Plasmas 5 (1998) 4337.

[10] R.L. Berger et al., Theory and three-dimensional simulation of light filamentation. Phys. Fluids B 5 (1993) 2243.

[11] C. Besse, N.J. Mauser and H.P. Stimming, Numerical study of the Davey-Stewartson System. ESAIM: M2AN 38 (2004) 1035-1054.

[12] H. Brezis, F. Golse and R. Sentis, Analyse asymptotique de l'équation de Poisson couplée à la relation de Boltzmann. Quasineutralité dans les plasmas. Note C. R. Acad. Sci. Paris Sér. I 321 (1995) 953-959.

[13] F. Castella, B. Perthame and O. Runborg, High frequency limit of the Helmholtz equations, II. Source on a manifold. Comm. Partial Differential Equations 27 (2002) 607-651.

[14] F.F. Chen, Introduction to Plasmas Physics. Plenum, New York (1974).

[15] M. Colin and T. Colin, On a Quasilinear Zakharov system describing Laser-Plasma Interaction. Differential Integral Equations 17 (2004) 297-330. 
[16] M. Colin and T. Colin, Cauchy problem and numerical simulation for a quasi-linear Zakharov system. Accepted for publication in Nonlinear Analysis.

[17] F. Collino, Perfectly matched absorbing layers for the paraxial equation. J. Comput. Phys. 131 (1997) 164-180.

[18] A. Decoster, Fluid equations and transport coefficient of plasmas, in Modelling of collisions. P.-A. Raviart Ed., Masson, Paris (1997).

[19] S. Desroziers, Modelisation de la propagation laser par résolution de l'équation d'Helmholtz, CEA internal report (2005).

[20] M. Doumic, F. Golse and R. Sentis, Propagation laser paraxiale en coordonnées obliques: équation d'advection-Schrödinger. Note C. R. Acad. Sci. Paris Sér. I 336 (2003) 23-28.

[21] M. Doumic, F. Duboc, F. Golse and R. Sentis, Numerical simulation for paraxial model of light propagation in a tilted frame: the advection-Schrödinger equation. CEA internal report (2005), preprint.

[22] M.R. Dorr, F.X. Garaizar and J.A. Hittinger, Simuation of laser-plasma filamentation. J. Comput. Phys. 17 (2002) $233-263$.

[23] V.V. Eliseev, W. Rozmus, V.T. Tikhonchuk and C.E. Capjack, Phys. Plasmas 2 (1996) 2215 and Phys. Plasmas 3 (1996) 3754 .

[24] M.D. Feit and J.A. Fleck, Beam nonparaxiality, filament formation. J. Opt. Soc. Amer. B 5 (1988) 633-640.

[25] F.G. Friedlander and J.B. Keller, Asymptotic expansion of solutions of $\left(\Delta+k^{2}\right) u=0$. Comm. Pure Appl. Math. 5 (1955) 387.

[26] S. Hüller, Ph. Mounaix, V.T. Tikhonchuk and D. Pesme, Interaction of two neighboring laser beams. Phys. Plasmas 4 (1997) $2670-2680$.

[27] J.D. Jackson, Classical Electrodynamics. Wiley, New York (1962).

[28] H. Jourdren, HERA hydrodynamics AMR Plateform for multiphysics simulation, in Proc. of Chicago workshop on AMR methods (Sept. 2003). Springer Verlag, Berlin (2004).

[29] J.B. Keller and R.M. Lewis, Asymptotic Methods for P.D.E: The reduced Wave Equation. Research report Courant Inst. (1964); reprinted in Surveys Appl. Math. 1, J.B. Keller, W. McLaughlin, G.C. Papanicolaou, Eds. Plenum, New York (1995).

[30] J.B. Keller and J.S. Papadakis, Eds., Wave Propagation and underwater Accoustics. Springer, Berlin. Lecture Notes in Phys. 70 (1977).

[31] Y.A. Krastsov and Y.I. Orlov, Geometric optics for Inhomogeneous Media. Springer, Berlin (1990).

[32] W.L. Kruer, The Physics of Laser-Plasma Interaction. Addison-Wesley, New York (1988).

[33] D. Lee, A.D. Pierce, E.S. Shang, Parabolic equation development in the twentieth century. J. Comput. Acoust. 8 (2000) 527-637.

[34] P. Loiseau, O. Morice et al., Laser-beam smoothing induced by stimulated Brillouin scattering. CEA internal report (2005).

[35] P. Mounaix, D. Pesme and M. Casanova, Nonlinear reflectivity of an inhomogeneous plasma. Phys. Rev. E 55 (1997) $4653-4664$.

[36] J.S. Papadakis, M.I. Taroudakis, P.J. Papadakis and B. Mayfield, A new method for a realistic treatement of the sea bottom in parabolic approximation. J. Acoust. Soc. Amer. 92 (1992) 2030-2038.

[37] G.C. Papanicolaou, C. Sulem, P.L. Sulem and X.P. Wang, Singular solutions of the Zaharov equations for Langmuir turbulence. Phys. Fluids B 3 (1991) 969-980.

[38] D. Pesme, Interaction collisionnelle et collective (Chap. 2) in La fusion par Confinement Inertiel I. Interaction laser-matière. R. Dautray-Watteau Ed., Eyrolles, Paris (1995).

[39] D. Pesme et al., Fluid-type Effects in the nonlinear Stimulated Brillouin Scatter, in Laser-Plasma Interaction Workshop at Wente, L. Divol Ed., Lawrence Livermore Nat. Lab. report UCRL-JC-148983 (2002).

[40] G. Riazuelo and G. Bonnaud, Coherence properties of a smoothed laser beam in a hot plasma. Phys. Plasmas 7 (2000) 3841.

[41] H.A. Rose, Laser beam deflection. Phys. Plasmas 3 (1996) 1709-1727.

[42] Shao et al., Spectral methods simulations of light scattering. IEEE J. Quantum Electronics 37 (2001) 617.

[43] G. Schurtz, Les codes numériques en FCI (Chap. 13), in La fusion par Confinement Inertiel, III. Techniques exp. et numériques, R. Dautray-Watteau Ed., Eyrolles, Paris (1995).

[44] W.W. Symes and J. Qian, A slowness matching eulerian method. J. Sci. Comput. 19 (2003) 501-526.

[45] F.D. Tappert, The parabolic equation approximation method, in Wave Propagation and underwater Accoustics, J.B. Keller and J.S. Papadakis Eds., Springer, Berlin. Lecture Notes in Phys. 70 (1977).

[46] V.E. Zakharov, Collapse of Langmuir waves. Sov. Phys. JETP 35 (1972) 908. 